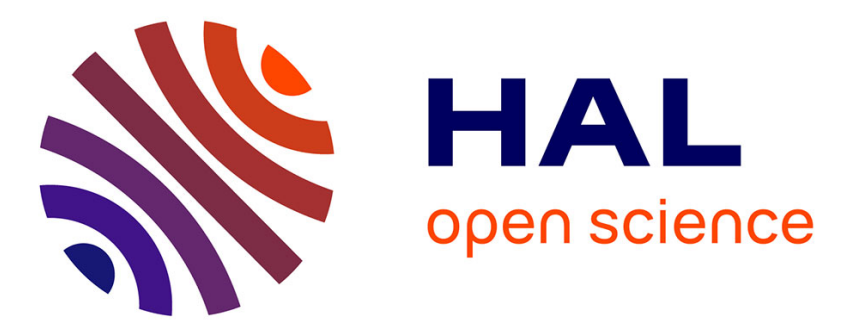

\title{
A stabilization process applied to a hidden variables method for evaluating the uncertainties on foundation impedances and their effect on vibrations induced by railways in a building
}

P. Ropars, Guy Bonnet, P. Jean

\section{To cite this version:}

P. Ropars, Guy Bonnet, P. Jean. A stabilization process applied to a hidden variables method for evaluating the uncertainties on foundation impedances and their effect on vibrations induced by railways in a building. Journal of Sound and Vibration, 2014, 333 (1), pp.1953-1971. 10.1016/j.jsv.2013.12.010 . hal-00932940

\author{
HAL Id: hal-00932940 \\ https://hal.science/hal-00932940
}

Submitted on 14 Jan 2016

HAL is a multi-disciplinary open access archive for the deposit and dissemination of scientific research documents, whether they are published or not. The documents may come from teaching and research institutions in France or abroad, or from public or private research centers.
L'archive ouverte pluridisciplinaire HAL, est destinée au dépôt et à la diffusion de documents scientifiques de niveau recherche, publiés ou non, émanant des établissements d'enseignement et de recherche français ou étrangers, des laboratoires publics ou privés. 


\title{
A stabilization process applied to a hidden variables method for evaluating the uncertainties on foundation impedances and their effect on vibrations induced by railways in a building
}

\author{
Pierre Ropars ${ }^{\mathrm{a}}$, Guy Bonnet ${ }^{\mathrm{a}}$, Philippe Jean ${ }^{\mathrm{b}}$ \\ a Université Paris-Est, Laboratoire Modélisation et Simulation Multi-Echelle, MSME UMR CNRS 2808, 5 bd Descartes, \\ 77454 Marne la Vallée Cedex 2, France

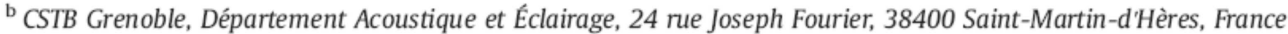

\begin{abstract}
A B S T R A C T
The paper is devoted to stochastic foundation impedance modeling for buildings submitted to vibrations. The hidden-variables method used in seismic engineering is revisited, due to a larger frequency range used in vibration prediction. Indeed, in this new context, instability of the solution and non-physical nature of mass and stiffness random matrices have been observed. The hidden variable method has been therefore implemented by enforcing explicitly the stability of the solution and the positiveness of mass and stiffness matrices. The effects of numerical parameters used throughout the process are shown and the improved hidden-variables method has been used for predicting the level of vibrations inside a building induced by railway sources. We present here steps of stabilization process, and then discuss on an example of application.
\end{abstract}

\section{Introduction}

Nowadays railways are still built near living areas and induce vibrations which are transmitted to buildings through foundation soils. The impact on the structure itself is rarely damaging, but vibrations can be felt badly by residents, and can induce structure-born sounds [1-4]. Therefore, it is important to predict the level of the vibrations induced into buildings, so that some convenient means can be introduced to reduce these vibrations, if necessary.

Studying this problem is still a hard task: it involves the description of the source, the transmission through the foundation soil, soil-structure interaction and finally, the transmission through the building. So, a convenient way to deal with these phenomena is to use sub-structuring, a technique which is frequently used to study soil-structure interaction in seismic engineering. The work which is presented in this paper is therefore an adaptation of a sub-structuring method well known of engineers in the field of vibrations, under the name of "mobility method". This method was initially conceived for studying the interaction between machines and their surrounding, but it can also be used for studying the noise coming from ground transmission. In the following, this method is translated by using the impedance instead of the mobility. So, the "mobility method" will be named thereafter "sub-structuring method". One main advantage of the method is to use more sophisticated means at each step of the computation. Note that the excitation source, here due to railway vibrations, is not considered in its full extent within this study. This is usually accounted for by considering a car-track-soil interaction problem and several models can be found in the literature [5,6]. The source is simplified in this study by using point forces whose level can be calibrated to account for experimental data.

From another point of view, a quick survey of the different parts to deal with when studying the overall problem of transmission of ground waves into buildings shows that an important point is a high level of randomness in the soil 
properties. So, this paper will show how the combination of the sub-structuring method and of an advanced method to describe random soil-structure interaction can allow to predict the statistical scattering of vibration amplitudes produced through a building.

The method, introduced previously in seismic engineering, uses a probabilistic model for any viscoelastic system containing a finite number of degrees of freedom (DOF) which are characterized by mass, damping and stiffness matrices $[7,8]$ and rests on a sound physical basis, i.e. maximal entropy principle. However, the infinite soil domain is not a priori characterized by a finite number of DOFs. So, the method used for the construction of a "random impedance" proceeds in two steps: in a first step, a "hidden structure" is built which, when coupled to the building, reproduces the response of the soil impedance. The degrees of freedom of such a hidden structure are called the "hidden variables". Next, the probabilistic model for a structure made up of a finite number of DOFs can be used.

The "hidden variables" method is based on the "lumped-parameter" model [9] containing a few connected degrees of freedom. This allows to approximate the dynamic stiffness by a rational fraction of $i \cdot \omega, \omega$ being the radial frequency. As a consequence, the method was also named as "rational approximation" of the dynamic stiffness (or its inverse). The main advantage of this kind of models was initially to replace the full definition of the impedance, which is often numerically computed at numerous given discretization points, by the approximated dynamic stiffness which is defined by a small number of parameters. The parameters of these models, being not too numerous, were initially obtained by using a simple least squared method. It was recognized early [10] that the dynamic stiffness must comply with a necessary stability condition related to the sign of the real part of the poles of the dynamic stiffness, as recalled thereafter. The following step in the approximation of the impedance by a reduced number of parameters was to consider more generally the approximation of the dynamic stiffness by the most general rational approximation [11]. An example of such a rational approximation is the case when the soil is replaced by a "hidden structure" characterized by its mass, damping and stiffness matrices $[12,13]$ considered as "hidden variables". Recent works in the context of rational approximations have been reviewed recently by Du and Zhao [11]. These authors present a state of the art of the different problems related to such a method, including stability conditions, resonance phenomena of the finite structure and different kinds of identification methods. The authors present new developments in relation to these different elements of the method. The reader can refer to this paper for more information about the state of the art related to this method in the domain of seismic engineering. It is worthwhile mentioning that, at the end of their literature review, these authors state: "the stability and identification for rational approximations of frequency response function of unbounded soil require a thorough study".

The previously cited works are related mainly to the field of seismic engineering. However, the main purpose of the present paper is to extend these methods to the field of vibration engineering for which the frequency range is larger. From our numerical experiments, this new context makes the identification of the parameters of the rational approximation more arduous. More particularly, it was observed that the matrices characterizing the "hidden structure" were not positive, which is not physically satisfying. It does not seem that such a problem was encountered in seismic engineering. Obviously, enforcing the positivity of these matrices must be effected simultaneously with the already known stability conditions, as shown thereafter. So, this paper presents an application of the "hidden variables" method to vibration engineering, including the modifications to introduce into the stabilization process in order to deal with this new context.

So, after a description of the main features of the sub-structuring method in Section 2, Section 3 is devoted to the description of the reproduction of the source impedance by using hidden variables in the simplest case, the so-called "stable case". Section 4 recalls quickly the probabilistic model which is used for building a "stochastic impedance" on the basis of the hidden variables model. In Section 5, it is emphasized that the stability is not always achieved and the section is devoted to describing a method which allows to deal with unstable cases. An example of unstable case is also presented, allowing to study the influence of the algorithm parameters. Finally, Section 6 shows an example of application to a multi-story building.

\section{Substructure modeling}

\subsection{Context of the substructure modeling}

The use of a sub-structuring method under the name of "mobility method" to study structure-borne sound has been known for a long time. It was used at the beginning for specifying the injected power coming from a vibratory machine into a structure [14-16]. Its principle consists in splitting the system under study into two sub-systems, a source and a receiver, which are more easily studied than the global system. The calculation of the power transmitted between both subsystems is based on the characterization of contact velocities and contact forces which are obtained from the impedances of both sub-systems. This method allows to study the influence of different types of waves and different components of displacement on the injected power [17-19]. In addition, it allows the comparison of different source systems by using the source descriptor $[14,20]$. In a more general case, the known impedances can be used for obtaining the power which is injected into different subsystems, when coupled to the same source.

In the following, the sub-structuring method is used for predicting the vibrations transmitted to a building. The substructuring comprises

- the "source" system which is composed of the set "ground + foundation", excited by the vibrations coming from a railway track located at the free surface of the soil.

- the "receiver" system which is the building excited by vibrations coming from its foundations. 
It should be noted that, for clarity of the paper, only the impedance $Z$, as defined in seismology, is used. The correspondence with the mobility $Y$ used in previously cited papers is simply $Y=\mathrm{j} \omega Z^{-1}$.

\subsection{Principle of the sub-structuring method}

The impedances $\mathbf{Z}_{R}$ and $\mathbf{Z}_{S}$ of both subsystems are obtained by computing the velocity induced by a given harmonic unit source applied to each subsystem. Then, the contact velocity and the contact force which are produced when the source is excited are provided by $[21,22]$

$$
\begin{gathered}
\mathbf{f}_{\mathbf{c}}=-j \mathbf{Z}_{\mathbf{R}}\left[\omega\left(\mathbf{Z}_{\mathbf{S}}+\mathbf{Z}_{\mathrm{R}}\right)\right]^{-1} \mathbf{Z}_{\mathrm{S}} \mathbf{v}_{\mathbf{L}} \\
\mathbf{v}_{\mathbf{c}}=\left(\mathbf{Z}_{\mathbf{S}}+\mathbf{Z}_{\mathbf{R}}\right)^{-1} \mathbf{Z}_{\mathrm{S}} \mathbf{v}_{\mathbf{L}}
\end{gathered}
$$

where $\mathbf{Z}_{\mathrm{S}}$ and $\mathbf{Z}_{\mathrm{R}}$ are the squared matrices of dimensions $n \times n$. $n$ is the overall number of DOFs at the interface. It is usually possible to split these DOFs into different small contact areas, each contact area behaving as a rigid surface, i.e. containing $n_{\text {dof }}$ degrees of freedom ( $n_{\text {dof }}$ being 3 for $2 \mathrm{D}$ cases and 6 for 3D cases). Then, the overall number of DOFs is given by $n_{\text {dof }} \times n_{c}$ where $n_{c}$ is the number of contact areas.

It is worthwhile mentioning that this formalism requires for the impedances to be of the same size when viewed from source or receiver. From another point of view, they must be causal and symmetric $[23,24]$.

\subsection{Source-system: free velocity and impedance}

Fig. 1 displays a typical situation where a source of excitation is applied to the soil surrounding the building and to the underground foundations. This set "source+soil + foundation" is treated as the source system. The extension of the soil zone to be taken into account for modeling the vibrations can be very large and the modeling of this zone is effected by the boundary element method in dynamic elasticity. The foundation itself has a limited extension and can be modeled by the finite element method. The source system is therefore modeled by a code coupling finite elements and boundary elements (named "Mefissto" $[25,26]$ ) which was built specifically for studying soil structure interaction. Of course, the use of such codes coupling finite element with boundary elements is now common for studying wave propagation problems [27-29].

The size of the impedance matrices depends on the number of DOFs at the interface between the source and the receiver. In order to reduce the size of these matrices, we can remark that the vibration frequencies induce wavelengths which are usually large (between several meters and several decimetres) compared to the elements at the interface between source and receivers, thanks to the choice of these elements (typically $20 \mathrm{~cm}$ for a separation between the source and the receiver introduced at the level of walls above the foundation). In this case, a surface element between the source and the receiver is small compared to the wavelengths related to waves transmitted along the walls of the building. This induces that the plane area of the section does not deform during the movement. This assumption is valid for frequencies up to $250 \mathrm{~Hz}$, which is the highest level of vibrations studied in this paper.

Finally, the number of degrees of freedom at the interface source-receiver is $6 \times N_{f}$ in three dimensions and $3 \times N_{f}$ in two dimensions, where $N_{f}$ is the number of foundations considered as contact areas.

Studying the source system makes it possible to obtain the "free velocity", as defined previously, and the source impedance. Some typical results on these quantities will be shown thereafter.

\subsubsection{Free velocity}

Fig. 1 displays how the free velocity is obtained numerically. The source system is excited by a given set of harmonic point forces having a unit amplitude, located at an excitation point close to the foundations and characterized by frequencies in the range [10-250] $\mathrm{Hz}$. All components of velocity on the top of the foundations are collected. They constitute the "free velocity", which will be used to produce contact velocities and contact forces at the interface.

Representing railway vibration sources by a point force may appear simplistic. However, despite its simplicity such point sources can be calibrated for reproducing the vibrations coming from in situ measurements [30]. In addition, more sophisticated sources can be obtained by superposing such point forces.

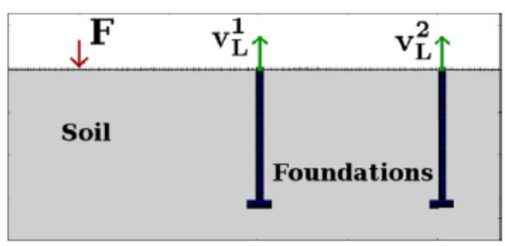

Fig. 1. Example of free velocity determination. 



\subsubsection{Source impedance}

There are four types of impedance components, each giving the ratio force over displacement:

- The direct component $Z_{x_{i} x_{i}}^{l l}$ giving the ratio at the same point and for the same DOF.

- The coupling component $Z_{x_{i} x_{j}}^{l l}$ giving the ratio at the same point but for different DOFs.

- The transfer component $Z_{x_{i} x_{i}}^{l_{k} x_{j}}$ giving the ratio at different points and for the same DOF.

- The last type $Z_{x_{i} x_{j}}^{l k}$ is the combination of the last two components and is called the transfer coupling component.

Obtaining the impedances of the source sub-system is performed by applying forces at the interface between the source and the receiver and by computing the induced kinematic degrees of freedom at the interfaces. In the case of $2 \mathrm{D}$ source subsystem, three kinds of excitations are applied at the top surface of the foundations, two for the components of the resultant and one for the moment. In each case three velocity components (two translations and one rotation) are obtained for each foundation and every component of the impedance is determined by

$$
Z_{x_{i} x_{j}}^{p q}=\mathrm{j} \omega \frac{F_{x_{i}}^{\text {foundation }_{p}}}{v_{x_{j}}^{\text {foundation }_{q}}}
$$

Finally, in the example of Fig. 2, the impedance matrices are $6 \times 6$ squared matrices. The results obtained from the numerical computation of the impedance can be checked easily against reciprocity relation involving symmetric terms of the impedance.

\subsection{Receiver system}

Several choices are possible for modeling the receiver system, for example the finite and/or boundary element method [31,32], the energetic methods [33] or wave methods [4,34]. A comparison of the results coming from different models can be found in [35]. For avoiding the discretization of the structure, a wave model based on the work of Mees and Vermeir [36] has been chosen.

This model uses a local description of simple elements, the assembly of its components allowing a global description of the structure. The elements of the structure in two dimensions are beams of finite length. They are described by EulerBernoulli equations. The dynamic equations comprise an equation for longitudinal displacement $u$ and an equation for flexure displacement $v$. The rotation is related to the transverse displacement by the usual relation of the beam theory $\alpha=\partial v / \partial x$, where $x$ is the space variable [21].

This method is very versatile concerning the situations which can be taken into account $[36,37]$.

In our example, there are $N_{e}$ levels supported by two exterior walls which are connected to two footings. The six boundary conditions at the footings are provided by imposing either velocity components or forces, as explained thereafter.

Having provided boundary conditions, the linear system of equations is solved numerically, providing the velocity at junctions, from which the velocity at each point of the structure can be deduced.

\subsubsection{Receiver impedance}

The impedance of the receiver is obtained by imposing a linearly distributed set of forces at one of the footings whose resultant (or moment) is given, all other components being free. All resulting velocity components are computed, producing the relevant components of the impedance matrix.

\subsubsection{Dynamic modeling of the structure}

When impedances and free velocity are known, the contact forces and velocities at the source-receiver interface with coupled the source and the receiver are determined by using relations (1) and (2) respectively.

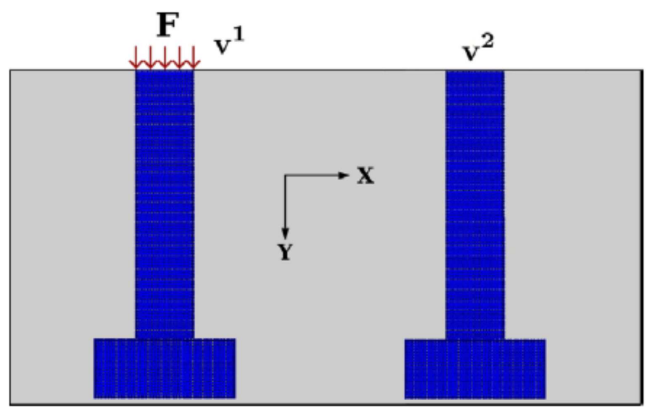

Fig. 2. Example of determination of the longitudinal source impedance. 
The dynamic modeling of the structure is achieved by enforcing the contact velocities at the footings of the structure. Finally, having obtained the distribution of particle velocities inside the structure, an indicator of the discomfort felt by the residents is obtained by computing the mean velocity on a slab given by $[38,39]$

$$
\langle\mathbf{v}\rangle=\frac{1}{N_{p}} \sum_{l=1}^{N_{p}}\left|\mathbf{v}\left(y_{l}\right)\right|
$$

where $N_{p}$ is the number of points, sufficient to properly describe the slab, and $y_{l}$ is related to the position of each point on the slab.

\section{Reproduction of the source impedance by a hidden variable system: the stable case}

\subsection{Generality}

As explained in Section 1, the source impedance provides the response of the set "soil + footings" to a given excitation applied at one of the footings. This is provided by the FEM+BEM code for a given set of physical parameters describing the soil behavior. Unfortunately, the data on soil properties are characterized by an important statistical scattering. This is well known by seismic engineers which take into account a wide range for the scattering of impedance values, using a coefficient of variation around 0.5 for the statistical scattering of the values of soil stiffness, from the experience of one of us.

So, with such a scattering, the study of the problem from a probabilistic point of view seems to be the most convenient. Providing directly scattered data on source impedance from the data coming from the mean model would be unfounded because such scattered data would miss basic features, being probably not causal. Producing scattered data for the impedance from direct modeling based on scattered values of soil properties is computationally too costly.

From another point of view, a well based probabilistic model for a system made up of a finite number of DOFs was provided by $[8,7]$. So, this section deals with the following problem: replace the soil impedance by a fictive structure (the socalled "hidden" structure) made up of a finite number of DOFs (the "hidden variables") which produces the same response as the soil-foundation system. Such a "hidden structure", reproducing the true soil+foundation system has obviously a causal response. In addition, all random "hidden structures" produced by using random values of the mass, damping and stiffness are also physically sound and respect the causality principle. It will be therefore used in the following section for building a physically sound stochastic model.

This kind of procedure was introduced previously in the field of seismic engineering by Cottereau et al. [12,13]. The present section presents succinctly the method as it was introduced by these authors. The improvements which are needed for the wider frequency range encountered in vibration engineering will be presented in Section 5. Let us now consider the set $\Gamma$ of all foundations containing $n_{\Gamma}$ DOF and connected to a "hidden structure" $\Omega$ containing a finite set of $n_{\Omega}$ DOFs. One considers now the overall impedance $\mathbf{S}$ of the whole system $\Gamma+\Omega$. It can be split as follows:

$$
\mathbf{S}=\left[\begin{array}{ll}
\mathbf{S}_{\boldsymbol{\Gamma}} & \mathbf{S}_{\mathbf{c}} \\
\mathbf{S}_{\mathbf{c}}^{T} & \mathbf{S}_{\Omega}
\end{array}\right]
$$

where $\mathbf{S}_{\Gamma}$ is a squared matrix of order $n_{\Gamma}, \mathbf{S}_{\Omega}$ a squared matrix of order $n_{\Omega}$ and $\mathbf{S}_{\mathbf{c}}$ a matrix of dimensions $n_{\Gamma} \times n_{\Omega}$.

The "hidden variables" impedance $\mathbf{Z}$ of $\Omega+\Gamma$ is the reduction of the matrix $\mathbf{S}$ on the set of the DOFs of $\Gamma$, and is given by

$$
\hat{\mathbf{Z}}=\mathbf{S}_{\Gamma}-\mathbf{S}_{\mathbf{c}} \mathbf{S}_{\Omega}^{-1} \mathbf{S}_{\mathbf{c}}^{T}
$$

The impedance of the overall system can be computed from the mass, damping and stiffness by

$$
\mathbf{S}=(\mathbf{j} \omega)^{2} \mathbf{M}+\mathbf{j} \omega \mathbf{D}+\mathbf{K}
$$

Each one of the matrices $\mathbf{M}, \mathbf{D}, \mathbf{K}$ can be split into sub-matrices in the same way as for $\mathbf{S}$. It is worthwhile noticing that all coefficients of $\mathbf{S}$ are second-order polynomials in $\mathbf{j} \omega$. With these notations, the problem is clearly to find a matrix $\mathbf{S}$ whose coefficients are all second-order polynomials in $\mathrm{j} \omega$, such that the related value of the "hidden variables" impedance $\hat{\mathbf{Z}}$ produces a satisfying approximation of $\mathbf{Z}$. This can be considered as an optimization problem, as explained in the following section.

\subsection{A related optimization problem and its solution}

\subsubsection{Introducing the optimization problem}

From Eq. (12), and from the definition of the inverse of $\mathbf{S}_{\boldsymbol{\Omega}}$ it can be seen that the approximate impedance $\hat{\mathbf{Z}}$ has the form $\hat{\mathbf{Z}}=\mathbf{N}(\omega) / q(\omega)$ where $q(\omega)$ has the degree $n_{q}=2 n_{\Omega}$ of the determinant of $\Omega$ and where all coefficients of $\mathbf{N}(\omega)$ have the degree $n_{q}+2$. So, having fixed the number of hidden variables and therefore the value of $n_{q}$, a first operation for building $\mathbf{Z}$ is to seek a polynomial matrix $\mathbf{N}$ and the polynomial $q$ having the previously defined degrees and such that $\mathbf{N} / q$ is as near as possible to the given impedance $\mathbf{Z}$. This is an optimization problem which is formalized by using the cost function $\varepsilon$ defined by

$$
\varepsilon=\sum_{l=1}^{L} w^{2}\left(\omega_{l}\right)\left\|\mathbf{N}\left(\omega_{l}\right)-q\left(\omega_{l}\right) \mathbf{Z}\left(\omega_{l}\right)\right\|_{F}^{2}
$$


in which the function $w$ is a weighting function which avoids numerical singularities, $\omega_{l}$ constitutes a set of frequencies at which the impedance $\mathbf{Z}$ is computed and $\|\cdot\|_{F}$ specifies the Frobenius norm. The weighting function is typically taken on the form $w(\omega)=|q(\omega)|^{q w}[40]$ and a study of its impact on the efficiency of the method is discussed in Section 5.4.

\subsubsection{Solving the optimization problem by using a specific metric space}

The number of coefficients to be obtained from the solution of the optimization problem becomes significant as soon as the number of hidden variables increases. So, the minimization of the cost function $\varepsilon$ is sought by using two means:

- The representation of the cost function as the product of a specific norm applied to the couple $(\mathbf{N}, q)$ and the construction of a scalar product which is compatible with this norm.

- A specific base of the finite dimension vector space of all possible couples $(\mathbf{N}, q)$, which is orthogonal with respect to the scalar product.

This allows to find an orthogonal basis $\boldsymbol{\Phi}$ from which the cost function is given by (see Appendix A)

$$
\varepsilon=\|\mathbf{V}\|_{W}^{2}=\left(\sum_{i=1}^{m h} \alpha_{i} \phi_{\mathrm{i}}\right)^{2}=\sum_{i=1}^{m h} \alpha_{i}^{2}
$$

where $\mathbf{V}$ is the vector $(\operatorname{vect}(\mathbf{N}), q)^{T}$ containing all components of $\mathbf{N}$ plus the values of $q, \alpha_{i}$ are its coordinate along the $i$ th vector of the basis $\boldsymbol{\Phi}$ and $m h$ is the number of vectors $\phi$.

\subsubsection{A first solution of the optimization process}

The solution of the minimization problem can now be written as a weighted sum of these vectors in which the coefficients are such that the cost function is minimum. The case $\alpha_{i}=0$ for all $i$ is obviously undesirable and it means that at least one of the $\alpha_{i}$ must be different from zero. Cottereau [12] proposed the following solution:

$$
\left\{\begin{array}{l}
\alpha_{i}=0 \quad \forall i<m h \\
\alpha_{m h}=1
\end{array}\right.
$$

In this process, all components of $(\mathbf{N}, q)$ on the orthogonal basis are set to zero, except the projection on the last vector of the basis. The solutions which are produced in such a way must comply with some specific conditions which will be defined in Section 5. These conditions are called thereafter "stability conditions". When the frequency range, the number of DOFs and/ or the number of hidden variables are not too large, the solution given by (10) complies with stability conditions. In some cases, it is necessary to take a few coefficients $\alpha_{i}$ equal to 1 to meet the stability conditions. For the complete frequency range and for the number of DOFs present in building vibrations, these conditions are not met with simple values of coefficients $\alpha_{i}$ and the technique of Section 5 must be used to enforce these conditions. At the present stage, and for the purpose of clarity, it is considered that a set of $\alpha_{i}$ is found which meets the stability conditions.

One can then proceed to the identification of the different matrices appearing in the overall impedance matrix $\mathbf{S}$. Knowing all components of $(\mathbf{N}, q)$ in the orthogonal basis, it is possible to construct all polynomials appearing in these quantities. At this stage, the objective becomes to build all components of the whole dynamic stiffness matrix $\mathbf{S}$.

\subsubsection{Identification of the dynamic stiffness matrix in the stable case}

When the solution of the minimization problem is obtained, one still needs the different sub-matrices which compose the matrices $\mathbf{M}, \mathbf{D}$ and $\mathbf{K}$ as $\mathbf{S}$ in (5). For clarity this step is described in Appendix B and the entire process can be found in [12].

It should be noted that the identification step requires the solution of a set of equations ((B.5) in Appendix B) which is overdetermined. This leads to consider that each eigenvalue of $\mathbf{S}_{\Omega}$ is counted $n_{\Gamma}$ times, increasing as a consequence the size of $\mathbf{S}$. Then the instabilities described in Section 5 increase with the number of DOFs considered in the initial impedance matrix Z.

This process leads finally to the matrices $\mathbf{M}, \mathbf{D}$ and $\mathbf{K}$ which define the properties of a "hidden structure" which produces an impedance close to the one which is calculated by using the mean properties of foundation soils.

\section{Construction of the stochastic impedance of the foundation}

The properties identified by using the "hidden structure" were obtained by using "mean" elastic properties of soils which are estimated at best by usual engineering ways. However, as underlined before, these values are affected by a strong statistical scattering and the values of $\mathbf{M}, \mathbf{D}$ and $\mathbf{K}$ can be considered as an estimation of the probabilistic mean of these quantities. The construction of a probabilistic model can be effected by several techniques like polynomial's chaos or fuzzy sets to describe parametric uncertainties. In addition, a modeling of the soil properties through stochastic elements may be used, as for similar studies performed for vibrations coming from road vehicles [41-47]. 
Here the choice has been made up of using a non-parametric stochastic model which is built by applying the maximum entropy principle to the dynamic impedance. This process avoids to solve numerous wave propagation problems related to multiple occurrences of soil properties. All possible realizations of mass, damping and stiffness matrices must have sound physical properties, being within a set of positive-definitive random matrices having convenient properties. The relevant theoretical considerations can be found in [7,8] and a short description of the process is given in Appendix C. The final result is that coefficients of matrices $\mathbf{M}, \mathbf{K}$ or $\mathbf{D}$ are governed by completely defined probability density functions which depend only on three scattering parameters $\delta_{M}, \delta_{C}, \delta_{K}$ (see Appendix C.2 and $[12,8]$ ).

\section{The stability problem and its solution}

The methodology described in Section 3 must be checked against physical properties of the system which is finally produced. The required physical conditions, or the so-called "stability conditions", are stated in a first step. Next, the solution for providing fully stable solutions is described. From a general point of view, the hidden variable method depends on two main parameters: the number of hidden variables and the weighting function introduced in the cost function (14). The influence of these two parameters on the solution of the stability problem is then studied.

\subsection{Description of the stability problem}

As mentioned above, the frequency range which must be accounted for when studying vibrations induced by railways is large, between 10 and $250 \mathrm{~Hz}$. This range is significantly larger than the one considered in seismic engineering problems. In these conditions, the structure of the computed impedance is significantly different from the one obtained in seismic engineering. The main consequence which was observed in numerous cases from the authors' experience is that the matrices built by using the hidden-variables method in its original form were not physically sound, meaning that they did not comply with some physical conditions denoted thereafter as "stability conditions".

The first kind of condition is imposed by the system of equations which relates the damping factors to the roots of polynomial $q[11,48]$. Roots of $q$ must have negative real parts (inducing a positive damping) and non-null imaginary parts. Such polynomials are called "Hurwitz". The second kind of condition requires that identified matrices $\mathbf{M}, \mathbf{D}$ and $\mathbf{K}$ are positive definite, i.e. that their eigenvalues are all positive. The "stability condition" is usually restricted to the first kind of condition. In this paper, "stability conditions" include by extension both kinds of conditions.

One way to produce polynomials $q$ which are Hurwitz is to add an additional constraint to the solution (10) of the polynomial $q$. This can be done through the Kharitonov theorem which gives a condition for a polynomial to be Hurwitz [49]. However, this method was shown to produce very often solutions which do not comply with the positiveness of matrices $\mathbf{M}, \mathbf{D}$ and $\mathbf{K}$. So, it was found that all conditions on $q, \mathbf{M}, \mathbf{D}$ and $\mathbf{K}$ had to be enforced simultaneously.

This is achieved by using a two-step process, the first step leading to a solution meeting all stability conditions and the second one minimizing the cost function.

\subsection{Solution of the stability problem}

\subsubsection{Step 1: Finding an acceptable solution}

To satisfy both previously defined conditions, the vector solution $\mathbf{P}$ must meet the following criteria:

$$
\begin{array}{ll}
\mathbf{P}=\sum_{i=1}^{m h} \alpha_{i} \boldsymbol{\phi}_{i}, & t q \\
\operatorname{Re}\left(z_{i}\right)<0, & 1 \leq i \leq n_{q} \\
\operatorname{Im}\left(z_{i}\right) \neq 0, & 1 \leq i \leq n_{q} \\
\min \left(\lambda\left(\mathbf{A}_{j}\right)\right)>0, & -2 \leq j \leq 0
\end{array}
$$

where $z_{i}$ denotes the $i$ th root of $q$ and $\lambda(\mathbf{A})$ the set of eigenvalues of the matrix $\mathbf{A}$. A is a global denomination for $\mathbf{M}, \mathbf{D}$ and $\mathbf{K}$.

From a numerical point of view, such conditions are not satisfactory, because they can produce solutions which may be considered as acceptable while being very close to unacceptable solutions. To circumvent this problem, more robust conditions are used, which are given by

$$
\begin{array}{ll}
\mathbf{P}=\sum_{i=1}^{m h} \alpha_{i} \boldsymbol{\phi}_{i}, & t q \\
\operatorname{Re}\left(z_{i}\right)<-\varepsilon, & 1 \leq i \leq n_{q} \\
\left|\operatorname{Im}\left(z_{i}\right)\right|>\varepsilon, & 1 \leq i \leq n_{q} \\
\min \left(\lambda\left(\mathbf{A}_{j}\right)\right)>\varepsilon, & -2 \leq j \leq 0
\end{array}
$$

where $\varepsilon$ is a small positive constant. 
All these conditions can be collected in a single function, producing a "distance" to the domain of acceptable solutions under the form

$$
\begin{gathered}
\mathbb{R}^{2\left(n_{q}+1\right)+3} \longrightarrow[0,+\infty[ \\
(x) \longrightarrow d(x) \\
d(x)=\left(\left(\operatorname{Re}\left(z_{1}\right)-\varepsilon\right)^{2} H\left(\operatorname{Re}\left(z_{1}\right)-\varepsilon\right)+\cdots+\left(\operatorname{Re}\left(z_{n_{q}}\right)-\varepsilon\right)^{2} H\left(\operatorname{Re}\left(z_{n_{q}}\right)-\varepsilon\right)\right. \\
\left.+\cdots+\left(\left|\operatorname{Im}\left(z_{n_{q}}\right)\right|-\varepsilon\right)^{2} H\left(\left|\operatorname{Im}\left(z_{n_{q}}\right)\right|-\varepsilon\right)+\cdots+\left(\lambda_{0}-\varepsilon\right)^{2} H\left(\lambda_{0}-\varepsilon\right)\right)^{1 / 2}
\end{gathered}
$$

where $\lambda_{i}(i=-2,-1,0)$ are the smallest eigenvalues of the identified matrices (reduced: $\mathbf{R}_{-2}, \mathbf{R}_{-1}$ and $\mathbf{R}_{\mathbf{0}}$ of Eq. (B.3) or global: $\mathbf{M}, \mathbf{D}$ and $\mathbf{K})$, and $H_{0}$ is the Heaviside function.

With this definition, any set of coefficients $\alpha$ which produces null values of $d$, or minimal values of a monotonic function of $d$, leads to an acceptable solution.

At the same time, it was found that it was convenient to minimize, instead of $d$, a monotonically decreasing function of $d$ with the form $f(d(\alpha))=-e^{-d(\alpha)}$, where $\alpha$ is a positive parameter.

It may be noticed that different values may be chosen for $\varepsilon$ in (13). However, it was observed that the use of different values of $\varepsilon$ in the different inequalities does not improve the results. Lastly, it was found to be more efficient for the minimization algorithm to enforce the different requirements in a sequence. We have tested only the sequential order related to the descending order in (13), while another order may be more efficient. The first one requires the condition on the roots of $q$. Then the second retains this condition and adds the requirement of semi-positiveness on matrices of order $n_{\Gamma}$. Finally the last step requires the positiveness of full matrices $\mathbf{M}, \mathbf{D}$ and $\mathbf{K}$. All these steps are performed by using Python's function: scipy.optimize.fmin, which is based on the downhill simplex algorithms.

Finally, if the process converges, a solution is found and can be optimized.

\subsubsection{Step 2: Optimization of the solution}

The optimization of the solution is performed by using again a constrained minimization procedure with the same (stability) conditions as above, but minimizing now the function described below in (14), which is directly related to the sum of the squares of the differences between estimated and initial components of the impedance. This leads to the set of equations and inequations

$$
\begin{gathered}
\mathbf{P}=\sum_{i=1}^{m h} \alpha_{i} \boldsymbol{\phi}_{i}, \quad t q \\
\boldsymbol{\alpha}_{\mathrm{opt}}=\arg \left(\min \left(\frac{\sum_{l=\mathrm{fmin}}^{\mathrm{fmax}}\left\|\mathbf{N}\left(\omega_{l}\right) / q\left(\omega_{l}\right)-\mathbf{Z}\left(\omega_{l}\right)\right\|_{F}^{2}}{\sum_{l=\mathrm{fmin}}^{\mathrm{fmax}}\left\|\mathbf{Z}\left(\omega_{l}\right)\right\|_{F}^{2}}\right)\right) \\
\operatorname{Re}\left(z_{i}\right)<-\varepsilon, \quad 1 \leq i \leq n_{q} \\
\left|\operatorname{Im}\left(z_{i}\right)\right|>\varepsilon, \quad 1 \leq i \leq n_{q} \\
\min \left(\lambda\left(\mathbf{A}_{\mathbf{j}}\right)\right)>\varepsilon, \quad-2 \leq j \leq 0
\end{gathered}
$$

This step has been performed by using Python's function scipy.optimize.cobyla, based on [50].

\subsection{Influence of algorithm parameters on the stability solution}

\subsubsection{Definition of the cases under study and of the degree of instability}

The cases presented here refer to foundations in concrete with footings which have $2 \mathrm{~m}$ depth in a soil having a stiffness corresponding to a "median soil" in the classification given by the French regulations on earthquake resistant constructions dated 1996 [51], for which shear wave velocity is $200 \mathrm{~m} / \mathrm{s}$. The geometry is the same as in Section 2 (Fig. 2) but the number of DOFs is two or three by foundation (two translations or two translations and one rotation respectively). In addition, the full impedance of both foundations is taken into account or only one foundation is considered. Finally, the number of DOFs is $2,3,4$ or 6 . In addition to the number of DOFs, the degree of $q$ lies between 2 and 16 . For all cases, the frequency range is [8-250] Hz with 485 discretized values. All independent components of the direct impedance are plotted in Fig. 9.

As explained before, the procedure of Section 3 produces directly stable solutions for simplest cases, while for more complex ones, the function defining the vector solution must be called several times during the previously defined "step 1" before reaching a stable solution. So, a natural degree of instability is the number of iterations, i.e. the number of computations of the vector solution before reaching convergence. The whole process depends on the coefficient of the weighting function and on the number of hidden variables. It also depends on the small positive constant $\varepsilon$ but this effect is not studied here.

In a first step, the effect of the weighting function on the degree of instability is studied.

\subsubsection{Effect of the coefficient of the weighting function on the degree of instability}

From the reference work [12], the value of $q w=-2$ seemed appropriated in the field of seismic engineering. However, in vibration studies, the frequency range is around ten times the one of seismic engineering. This feature affects directly the frequency effect and it was observed that such a weighting function coefficient can no more be used. The values of 
weighting function coefficients which were found to be efficient are lying between -1 and 1 . Using such a range for the weighting function coefficient, the degree of instability is reported in Fig. 3 as a function of the weighting function coefficient, for two values of the degree $n_{q}$ of $q$ (4 and 12) (which is two times the number of hidden variables).

The missing points indicate occurrences where the entire process fails and zero refers to a naturally stable case where no stabilization process is needed.

Fig. 5 is split into two parts. Indeed, the rotation is known as producing the more instabilities [52] and the cases involving rotation terms are drawn separately. A first remark is that the most stable region is different for each case. For example, when $n_{q}=2$, the case 2 is stabilized for $q w \in[0.2,1]$ and the case 1 for $q w \in[-1,1]$. Secondly, the degree of instability is higher when the number of hidden variables or the number of DOFs are increased. In addition, the results show that there is no directly stable case for $n_{q}=12$ hidden variables and that case 4 has no solution for 12 hidden variables.

To summarize, the minimum and maximum values for which a stabilized solution is found are shown in Table 1 . The optimum value $q w_{\text {opt }}$ is determined by the situation where the number of iterations is the smallest. As a conclusion, contrary to the case of seismic engineering, we cannot determine here a unique value of $q w$ which would be convenient in any case. This parameter must be adapted to each specific case.

\subsection{Effect of the number of hidden variables on the instability process}

Fig. 4 compares the number of iterations at step 1 needed for stabilization against the number of hidden variables for all four cases, the weighting function being chosen as $w=|q|^{q w_{\text {opt }}}$ defined above.

This figure shows that the number of iterations needed for stabilization depends on the numbers of DOFs and on the number of hidden variables (represented in the figures by the degree $n_{q}$ of polynomial $q$ ). It can be seen that the cases are mainly unstable: in numerous cases, it has been necessary to proceed to step 1 of stabilization.

It should also be noticed that cases 2 and 4 which involve the components of rotation of the foundation are more unstable. The terms related to rotation impedance are quite different from the translation terms (terms homogeneous with a momentum instead of a stiffness). This produces a bad conditioning of the impedance matrix, which affects all the process.
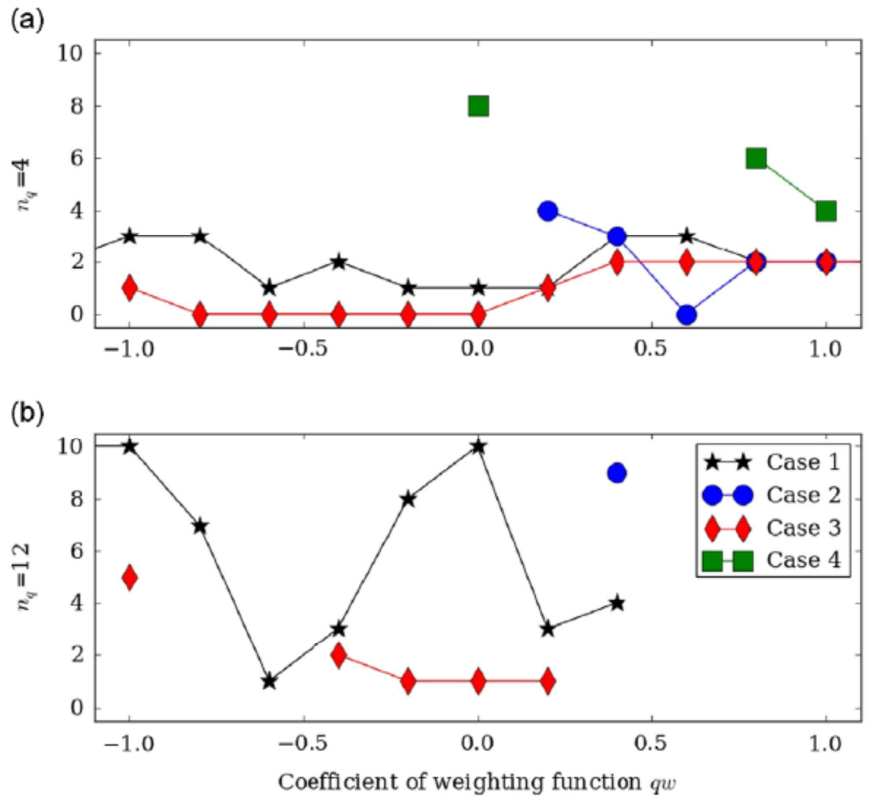

Fig. 3. Impact of the coefficient of the weighting function on the number of iterations in the identification process described in Section 5.2 .1 , for $n_{q}=4$ (a) and for $n_{q}=12$ (b); case 1 ( 2 DOFs) with star markers, case 2 (3 DOFs) with circular markers, case 3 (4 DOFs) with rhombus markers and case 4 ( 6 DOFs) with squared markers.

Table 1

Synthesis of the effect of $q w$ on the number of iterations needed for the stabilization.

\begin{tabular}{lclc}
\hline Case & $q w_{\min }$ & $q w_{\max }$ & $q w_{\text {opt }}$ \\
\hline 1 & -1 & 0.4 & -0.6 \\
2 & 0.4 & 0.4 & 0.4 \\
3 & -0.4 & 0.2 & -0.2 \\
4 & 0.8 & 1 & 1 \\
\hline
\end{tabular}




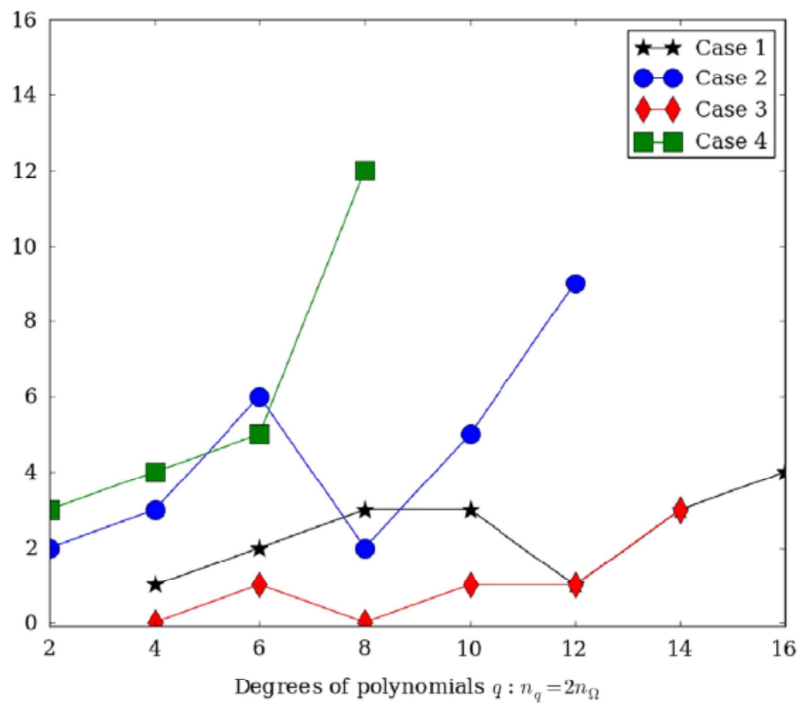

Fig. 4. Number of iterations at step 1 needed for obtaining stabilized solutions for situations described in Fig. 2 , case 1 ( 2 DOFs) with star markers, case 2 ( 3 DOFs) with circular markers, case 3 (4 DOFs) with rhombus markers and case 4 (6 DOFs) with squared markers.
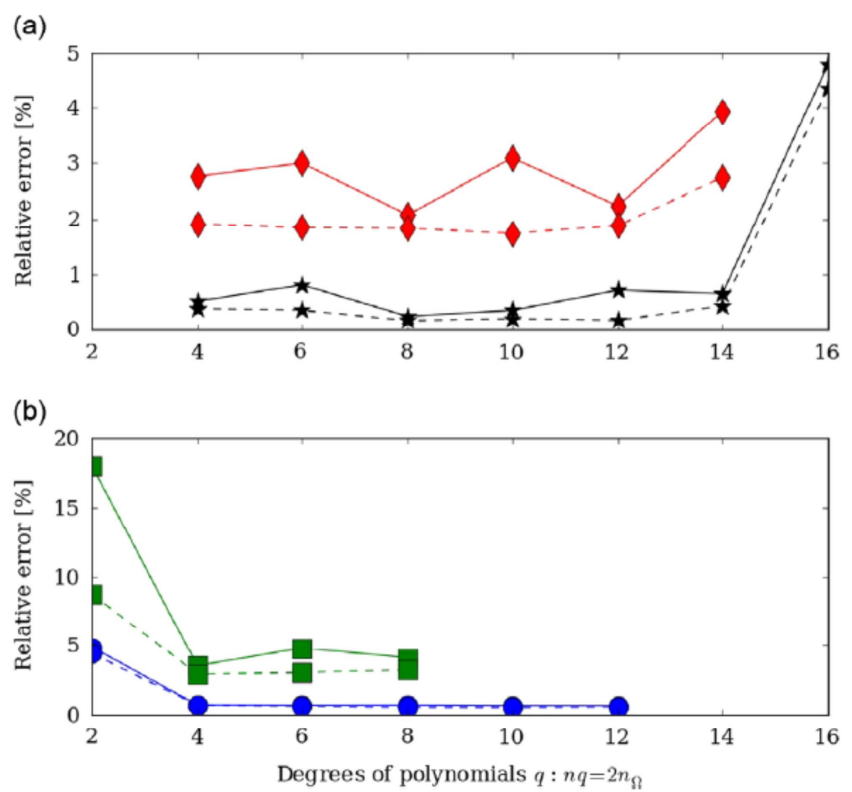

Fig. 5. Impact of the number of hidden variables and of the optimization phase on the relative error. The full lines show results without optimization phase (step 2 of our process) and dashed lines show results with optimization phase. Upper part (a): case 1 (2 DOFs) with star markers and case 3 (4 DOFs) with rhombus markers. Lower part (b): case 2 (3 DOFs) with circular markers and case 4 (6 DOFs) with squared markers.

The main trend is that the instability degree globally increases with the number of hidden variables with, in some cases, a local minimum for some values of $n_{q}$.

Next, another point of interest is the gain in accuracy obtained while increasing the number of hidden variables or when proceeding to the step 2 of the stability problem. To quantify the influence of these components on the overall procedure, the relative error after identification is provided. It is defined by

$$
\Delta \mathbf{Z}\left(n_{q}\right)=\frac{\sum_{l=\mathrm{fmin}}^{\mathrm{fmax}}\left\|\mathbf{N}\left(\omega_{l}\right) / q\left(\omega_{l}\right)-\mathbf{Z}\left(\omega_{l}\right)\right\|_{F}^{2}}{\sum_{l=\mathrm{fmin}}^{\mathrm{fmax}}\left\|\mathbf{Z}\left(\omega_{l}\right)\right\|_{F}^{2}}
$$

The effect of the number of hidden variables and of the optimization step can be seen in Fig. 5, where the relative error is given as a function of $n_{q}$ for all cases. The higher part of the figure is related to cases 1 and 3, while the lower part is related to cases 2 and 4 . In each figure the results are given by continuous lines without optimization step (i.e. after obtaining 


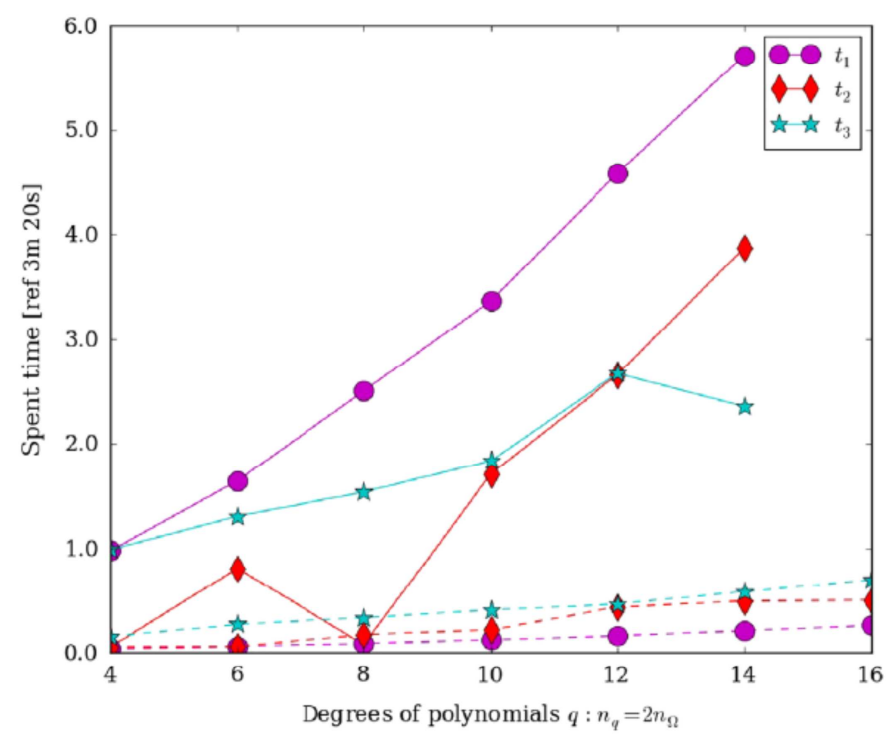

Fig. 6. Time duration for construction of base $\boldsymbol{\Phi}$ (circular markers), stabilization (rhombus markers) and optimization (star markers) for case 1 (dashed line) and case 3 (full line).

a stable solution) simultaneously with the ones obtained with optimization (dashed lines). The results show that increasing $n_{q}$ improves significantly the accuracy of the result up to $n_{q}=4$, but that a higher value of $n_{q}$ is without a significant effect on the accuracy of the result. For the optimization effect, it can be seen that the gain provided by this step is weak, except for $n_{q}=2$. In fact, the relative error is of the same order of magnitude with or without the optimization step for other values of $n_{q}$. This order of magnitude of the error seems to be governed mainly by the physical situation.

A final effect of the choice of the parameters is in the computation time spent during the process. This time can be split as follows:

- the different duration time for all steps of the procedure, i.e. calculating the basis $\boldsymbol{\Phi}$ (time $t_{1}$ ),

- the search for an acceptable solution (time $t_{2}$ ),

- the optimization of the solution (time $t_{3}$ ).

These different partial times are shown in Fig. 6 for cases 1 and 3.

These curves show that the number of hidden variables is strongly dependent on the case under study. Obviously, the time mainly increases with $n_{q}$. The increase of computation time of the basis $\boldsymbol{\Phi}$ is mainly due to the number of vectors of the basis which rises about as $n_{\Gamma}^{2}\left(2 n_{\Omega}+3\right)$.

Finally, it can be concluded that it is generally necessary to proceed to step 1 of the stability problem, because the solution obtained by using the process of Section 3 leads generally to unstable solutions. Under the condition of choosing conveniently the degree of the exponent $q w$ in the cost function, a value of $n_{q}=4$ produces an acceptable solution without a further optimization. The use of higher values of $n_{q}$ is more time consuming for a gain which is not significant.

\section{An application to a six-story building}

To illustrate the approach outlined so far, the case of a six-story building is studied in two dimensions. As mentioned earlier, the impedance of the source (foundation + soil) and the impedance of the receiver (structure without foundation) are characterized separately. The impedance of the source, as defined in Section 2.3.2, is used to obtain a set of realizations of the stochastic impedance. Both subsystems are then coupled with the sub-structuring method.

\subsection{Description of example}

The global system can be seen in Fig. 7, comprising a six-story building located at $10 \mathrm{~m}$ of a railway. The excitation is represented by a concentrated force applied to the boundary element which is located at the position of the railway in the FEM-BEM simulation of the free velocity. The structure is modeled by the wave model described above. The floors are uniform and are $2.8 \mathrm{~m}$ high and $10 \mathrm{~m}$ wide. All beams and foundations have a thickness of $0.2 \mathrm{~m}$. The material of the structure is concrete, its physical parameters are specific mass $\rho_{b}=2400 \mathrm{~kg} / \mathrm{m}^{3}$, Young's modulus $E_{b}=26 \mathrm{GPa}$, Poisson's ratio $\nu_{b}=0.3$ and damping factor $\eta_{b}=0.05$. The soil is constituted of one layer of $10 \mathrm{~m}$ depth, resting on an infinite stiffer 


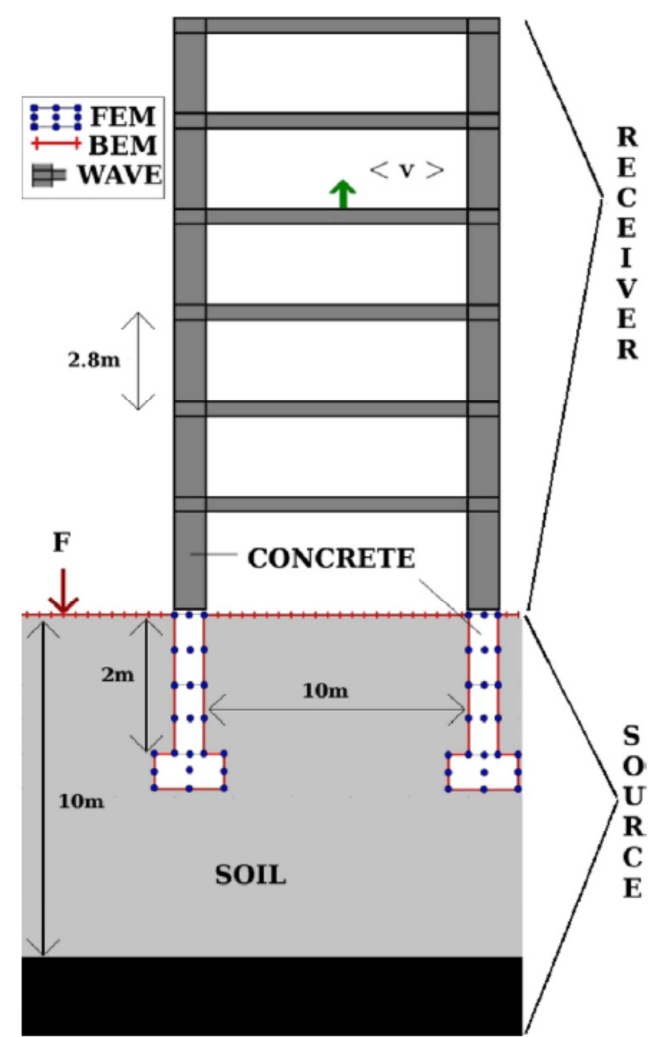

Fig. 7. Synthesis of the numerical features in use: soil modeled with boundary element, foundations with finite element and receiver by a wave approach.

domain, both modeled with BEM. The properties used for the soil are $\rho_{s}=1600 \mathrm{~kg} / \mathrm{m}^{3}, E_{s}=200 \mathrm{MPa}, \nu_{s}=0.257$ and $\eta_{s}=0.1$ and the properties of the stiffer domain are $\rho_{s}=3000 \mathrm{~kg} / \mathrm{m}^{3}, E_{s}=200 \mathrm{GPa}, \nu_{s}=0.25$ and $\eta_{s}=0.1$.

\subsection{Deterministic impedance}

The identified impedance which results in the process described in Section 3-5 is shown in Fig. 8. The system under study has two foundations and is studied in two dimensions, which induces an impedance of $4 \times 4$ components of translation (the rotation DOF is not considered here). The computation was performed with 7 hidden variables (i.e. $n_{q}=14$ ), the weighting function is $w(\omega)=|q(\omega)|^{0.4}$.

The identification error here is about 5 percent and the computation time on the order of $2 \mathrm{~h}$.

The initial impedance and the one obtained after identification are shown in Fig. 8(a)-(d). It can be seen that all components are well identified except terms in which oscillations occur (Fig. 8(b) and (e)). To better evaluate these components, the polynomial $q$ should have a degree superior to the number of oscillations. However, while the identification process is efficient with $n_{q}=28$ (less than 2 percent of error), the stabilization process fails in obtaining an adequate solution for such a large polynomial order.

\subsection{Stochastic impedance}

Stochastic impedance coefficients resulting from the procedure given in Section 3 and Appendix C are shown in Fig. 9. The scattering parameters around the deterministic values are given by $\delta_{M}=0.3, \delta_{K}=0.5$ and $\delta_{D}=0.5$.

The curves of the lower part of Fig. 9 display a large scattering, whose order of magnitude is obviously related to the scattering coefficients $\delta_{M}, \delta_{D}, \delta_{K}$.

\subsection{Stochastic floor level velocity}

The effect of the stochastic impedance on vibrations within the building is provided by computing the contact velocity $\mathbf{v}_{\mathbf{c}}$ at the interface between the source and the receiver, as seen in Section 2. This contact velocity being a function of the source impedance, it is therefore also random. The contact velocity is computed for each realization of the source impedance. This 
(a)

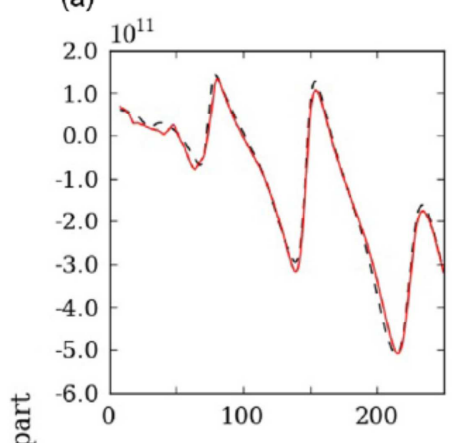

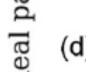

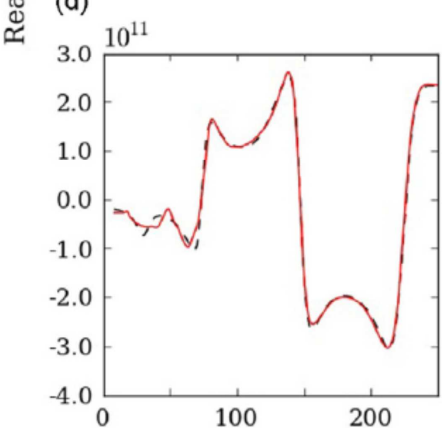

(a)

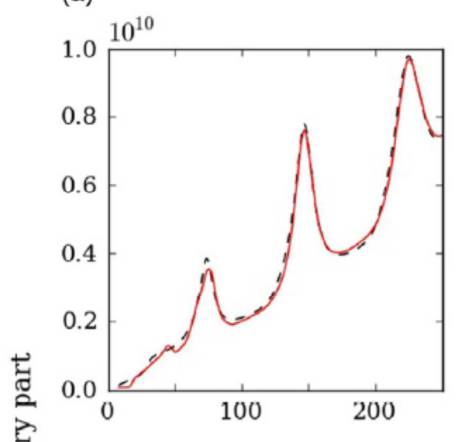

(d)

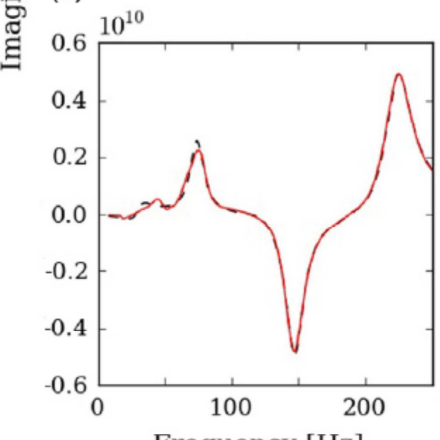

Deterministic impedance $[\mathrm{m} / \mathrm{N}]$

(b)

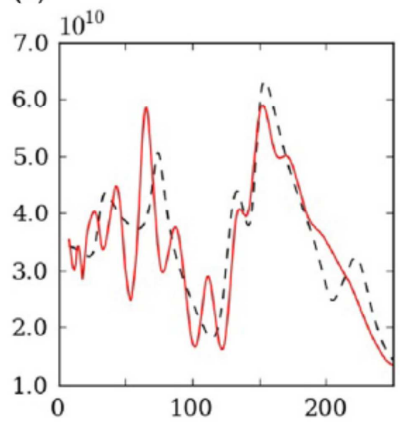

(e)

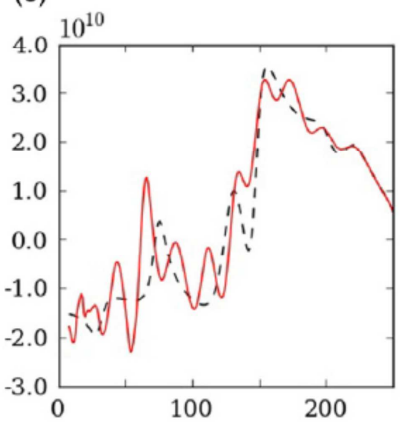

(b)

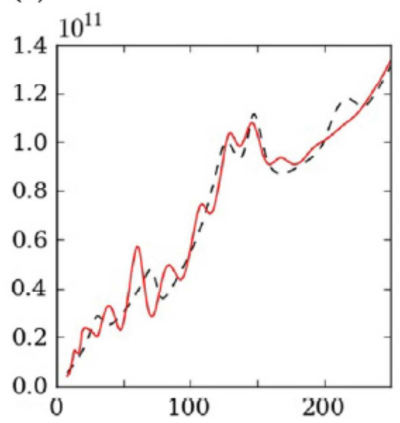

(e)

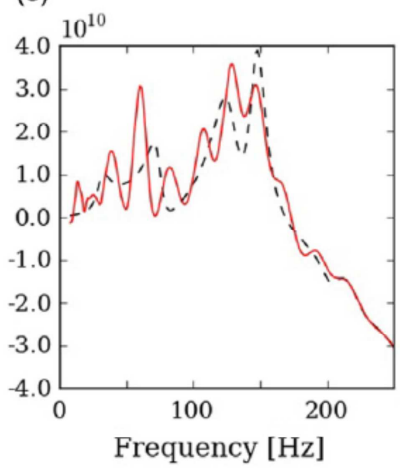

(c)

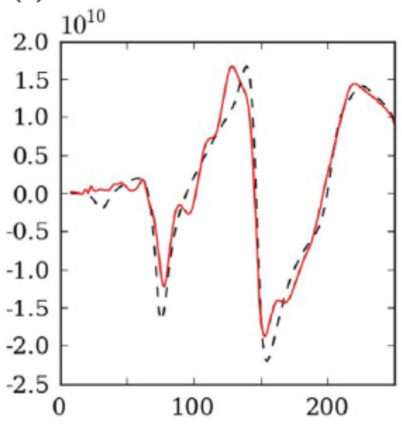

(f)

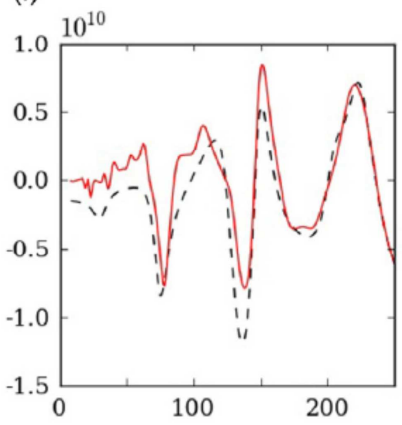

(c)

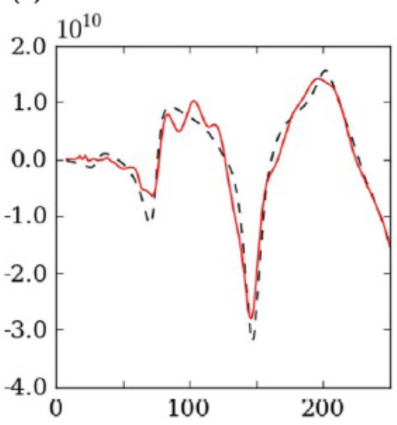

(f)

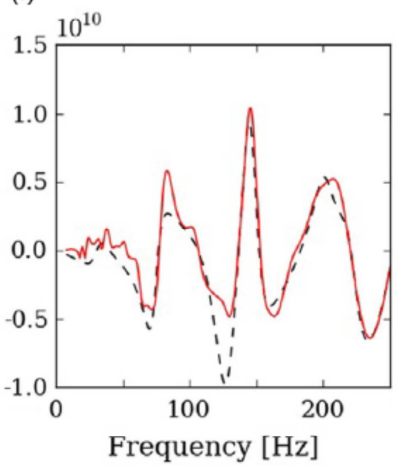

Fig. 8. At the top, initial (solid line) and identified (dashed line) components of impedance matrix, graphs (a), (b) and (c) display real parts of the three direct and coupling components $Z_{x x}^{11}, Z_{y y}^{11}$, and $Z_{x y}^{11}$ respectively and graphs (d), (e), and (f) correspond to real parts of the three transfer components $Z_{x x}^{12}, Z_{y y}^{12}$, and $Z_{x y}^{12}$ respectively. At the bottom, graphs (a), (b), (c), (d), (e), (f) display the imaginary part of the impedances. 
(a)
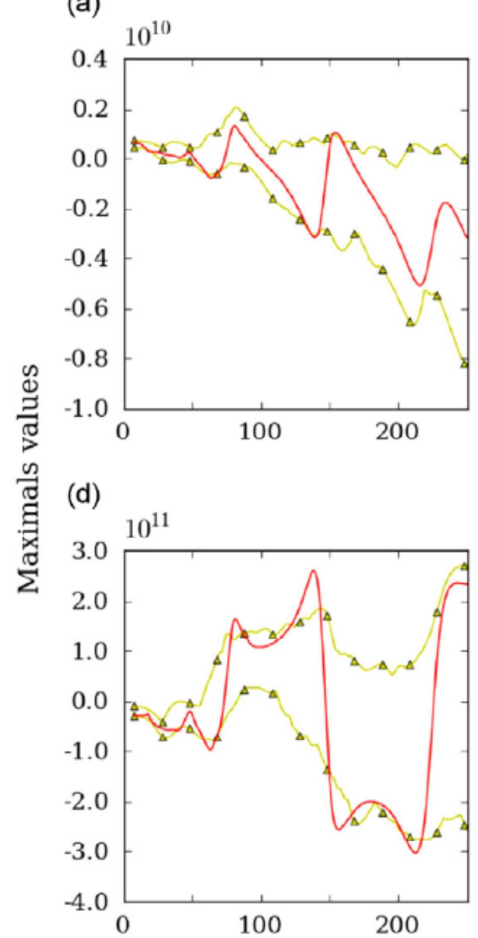

(a)

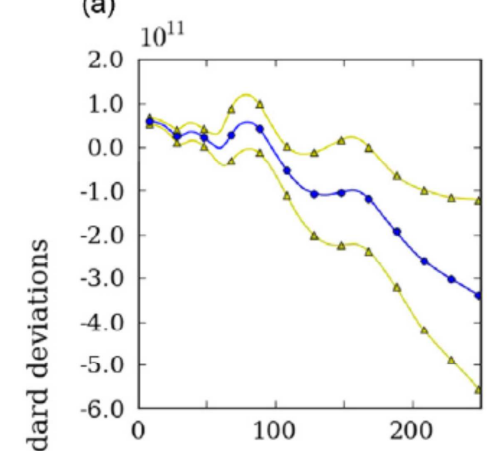

(d)

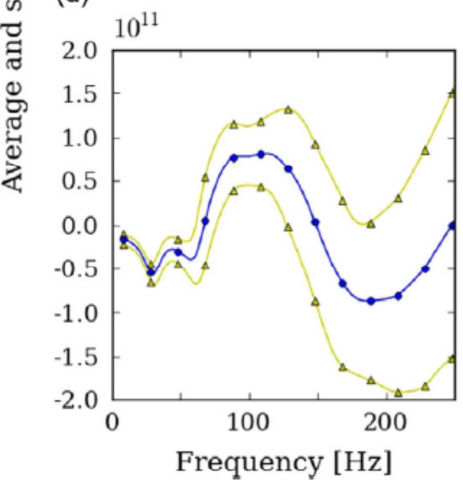

Stochastic impedance $[\mathrm{m} / \mathrm{N}]$

(b)

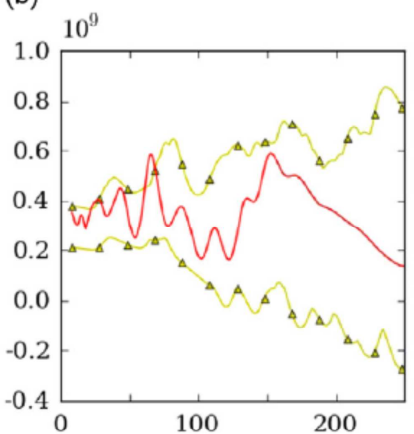

(e)

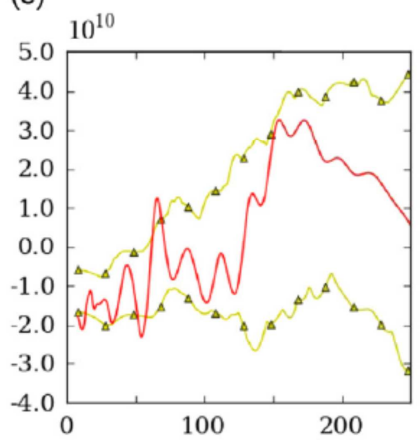

(b)

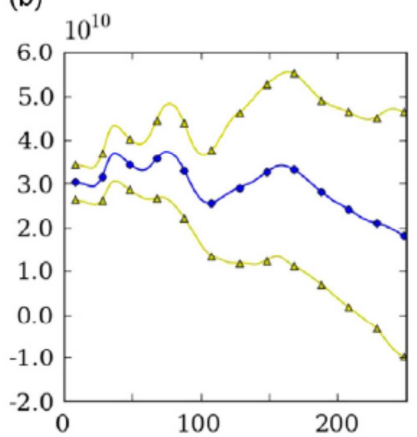

(e)

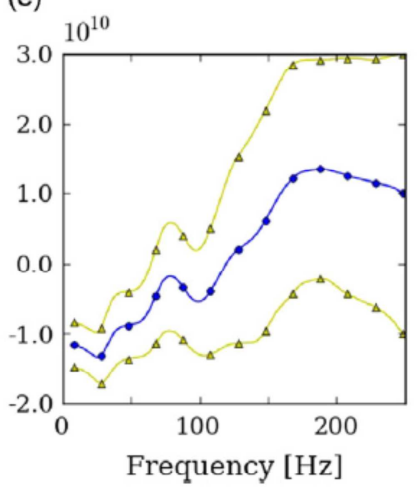

(c)

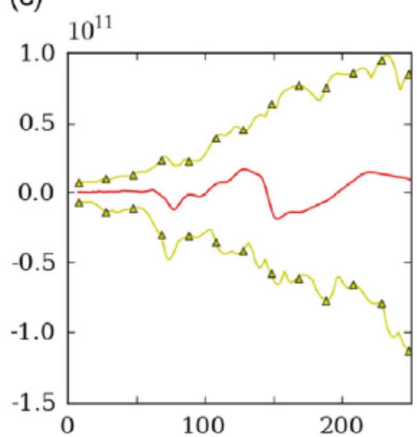

(f)

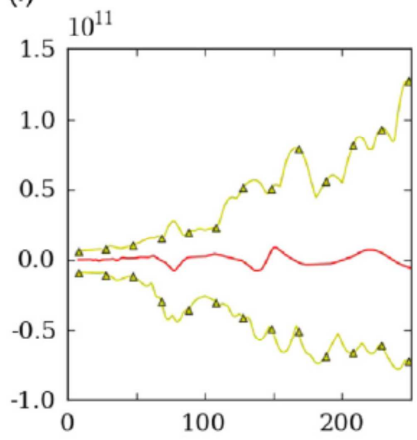

(c)

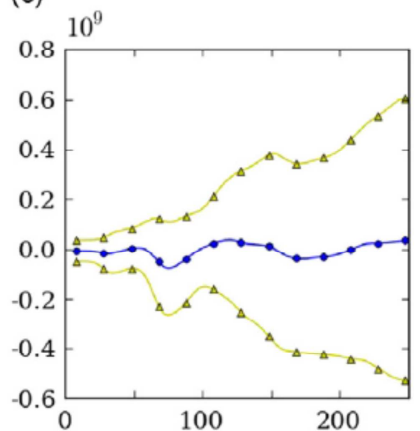

(f)

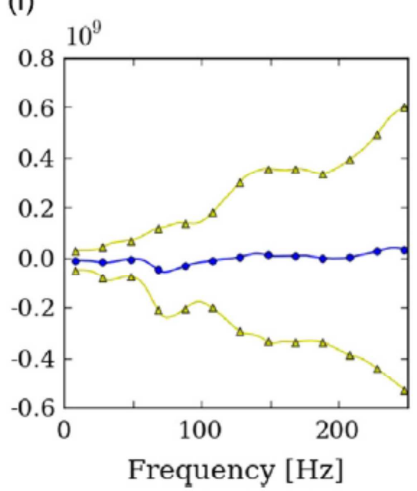

Fig. 9. At the top, initial impedance (solid line) and maximal values of stochastic realizations (triangular markers) components of impedance matrix, graphs (a), (b), and (c) display real parts of the three direct and coupling components $Z_{x x}^{11}, Z_{y y}^{11}$, and $Z_{x y}^{11}$ respectively and graphs (d), (e), and (f) correspond to real parts of the three transfer components $Z_{x x}^{12}, Z_{y y}^{12}$, and $Z_{x y}^{12}$ respectively. At the bottom, graphs (a), (b), (c), (d), (e), (f) display the original mean value (circular markers) and the range of variations (triangular markers) calculated with 10000 stochastic realizations. 

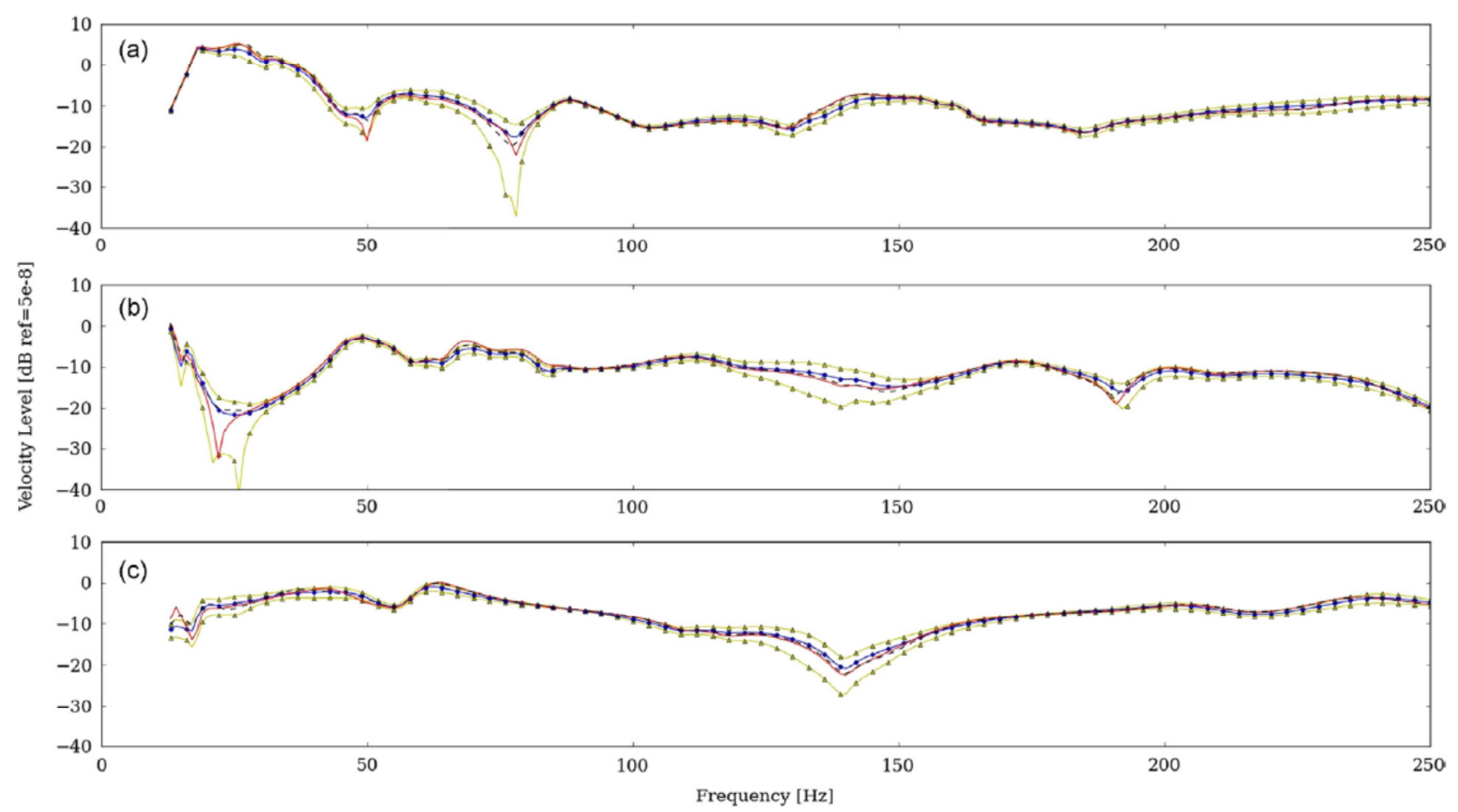

Fig. 10. Stochastic average velocity of the fourth slab structure, in solid line the initial velocity, in dashed line the identified velocity. Circular markers are related to the mean of stochastic occurrences and triangular markers to their standard variations: (a) vertical component, (b) horizontal component and (c) rotational component.

leads to a stochastic contact velocity of the form

$$
\begin{gathered}
\left.\overline{\mathbf{V}_{\mathbf{c}} i}=\overline{\mathbf{Z}_{\mathbf{S}_{i}}}\left(\overline{\left(\mathbf{Z}_{\mathbf{S}}\right.}+\mathbf{Z}_{\mathbf{R}}\right)^{T}\right)^{-1} \mathbf{v}_{\mathbf{L}} \\
\overline{\mathbf{V}}_{\mathbf{c}}=\left\{\overline{\mathbf{V}_{\mathbf{c} 0}}, \overline{\mathbf{V}_{\mathbf{c}}}, \ldots, \overline{\mathbf{V}_{\mathbf{c}}}, \ldots, \overline{\mathbf{V}_{\mathbf{c}}}\right\}
\end{gathered}
$$

where $N$ is the number of stochastic occurrences.

The wave model of the building is solved with each occurrence of the stochastic velocity, which produces a response (average velocity at different points) on a slab located inside the building. The results for all occurrences of the impedance produce the occurrences of the stochastic average velocity of the slab. This set is given in Fig. 10 for the fourth slab of the building.

This figure shows that the scattering on the induced velocities appears along all the frequency ranges. However, the local minimum of the induced velocity observed on the deterministic curve is strongly affected by the scattering, more specifically for the horizontal velocity of the slab at mean frequencies (around [15-35] Hz) and for all kinds of velocity at higher frequencies (between $125 \mathrm{~Hz}$ and $160 \mathrm{~Hz}$ ). In addition, the level of statistical scattering is globally lower than the one observed for the source impedances. It should also be noticed that if the scattering is important, the main tendency is to produce minima which are inferior by $10-20 \mathrm{~dB}$ to the mean (deterministic) value. However, if the difference between the mean value and the highest value obtained by scattering is considered, the difference is generally less than $10 \mathrm{~dB}$.

\section{Conclusion}

Using the method of sub-structuring allows to split the study of railway vibration transmitted to buildings into two parts: the study of the response of the foundations and the one of the structure. With this decoupling the impact on the building of the uncertainties related to the foundation impedance can be evaluated.

The method described in this paper evaluates the impact of uncertainties related to soil properties on the vibrations of the building structure. The procedure giving the average value of the impedance is limited neither by the number of degrees of freedom nor by the number of contact areas between the building and the foundations. The most difficult part of the work is to build a "hidden structure" which, when coupled to the building, reproduces the same response as the source containing simultaneously soil and foundation. The method is based on the construction of an orthogonal basis of functions in a hermitian space whose scalar product is built on the cost function. Such a method was used as such in seismic engineering, but needs some improvements, because the basic method can lead in numerous cases of vibration studies to physically unsatisfying solutions. The improvements described in the paper have been shown to be efficient. Finally the application of the method allows to build the stochastic response inside the building to a given excitation applied to the soil. 
Obviously, the method developed in this paper takes into account the scattering of results coming from the soil properties near the foundations, but does not account for the uncertainties coming from sound propagation through a soil characterized by randomly space varying soil properties. Taking into account this other kind of randomness would lead to model the soil properties along the propagation of the soil by finite elements instead of boundary elements, as effected here, in order to build random matrices to account for randomness of waves propagating through the soil. This last point will be the subject of a further study.

\section{Appendix}

Several steps on the hidden variables method, developed by Cottereau et al., are reported here for the clarity of the paper. Complete information can be found in Refs. $[7,8,12,13]$.

\section{Appendix A. Optimization problem}

As explained in Section 3.2.2, the optimization problem (8) can be written under a more convenient form. To quantify the component of the cost function it was found efficient to project it onto an orthogonal basis related to a conveniently chosen norm.

\section{A.1. Specific scalar product and norm applied to couples $(\mathbf{N}, q)$}

At each couple $(\mathbf{N}, q)$ one associates the column vector $\mathbf{V}(\omega)=\mathbf{V}(\mathbf{N}(\omega), q(\omega))=(\operatorname{vect}(\mathbf{N}), q)^{T}$, which contains all the $n_{T}^{2}$ coefficients of matrix $\mathbf{N}$ (stacked in vect( $\mathbf{N}$ ) column by column) plus the value of $q$. A norm is introduced into the set of all vectors $\mathbf{V}$ which are thus constructed by using a sesquilinear product $\left\langle\mathbf{V} \mid \mathbf{V}^{\prime}\right\rangle_{W}$ which is defined by

$$
\left\langle\mathbf{V} \mid \mathbf{V}^{\prime}\right\rangle_{W}=\operatorname{Re}\left(\sum_{l} w\left(\omega_{l}\right) \mathbf{V}^{\prime *}\left(\omega_{l}\right) \mathbf{W}\left(\omega_{l}\right) \mathbf{V}\left(\omega_{l}\right)\right)
$$

where $w\left(\omega_{l}\right)$ are the values obtained from the weighting function of $(8)$ and $\mathbf{W}\left(\omega_{l}\right)$ is the matrix given by

$$
\mathbf{W}\left(\omega_{l}\right)=\left[\begin{array}{cc}
\mathbf{I}_{\mathbf{n}_{\mathbf{r}}} & -\operatorname{vect}\left(\mathbf{Z}\left(\omega_{l}\right)\right) \\
-\operatorname{vect}\left(\mathbf{Z}\left(\omega_{l}\right)\right)^{*} & \left\|\mathbf{Z}\left(\omega_{l}\right)\right\|_{F}
\end{array}\right]
$$

The norm of a specific vector $\mathbf{V}$ is obtained by the usual relation $\|\mathbf{V}\|_{W}=\sqrt{\langle\mathbf{V} \mid \mathbf{V}\rangle_{W}}$.

\section{A.2. An orthogonal basis of the space of couples $(\mathbf{N}, q)$}

The couple $(\mathbf{N}, q)$ can be decomposed into the basis made up of all sub-matrices which contain only a monomial chosen among all coefficients of $(\mathbf{N}, q)$. This basis contains $m h=n_{\Gamma}^{2}\left(n_{q}+3\right)+\left(n_{q}+1\right)$ vectors, where $n_{q}$ is the degree of the polynomial $q$. Starting from this basis, using the vector product $\left\langle\mathbf{V} \mid \mathbf{V}^{\prime}\right\rangle_{W}$ and the Gram-Schmidt process, one can construct an orthogonal basis $\boldsymbol{\Phi}$ of the vector space containing all possible values of $(\mathbf{N}, q)$. This basis produces the expression (9) for the cost function:

$$
\varepsilon=\|\mathbf{V}\|_{W}^{2}=\left(\sum_{i=1}^{m h} \alpha_{i} \boldsymbol{\phi}_{i}\right)^{2}=\sum_{i=1}^{m h} \alpha_{i}^{2}
$$

where $\alpha_{i}$ is the coordinate of vector $\mathbf{V}$ along the $i$ th vector of the basis $\boldsymbol{\Phi}$ and $m h$ the number of vectors $\boldsymbol{\phi}$.

\section{Appendix B. Identification of the dynamic stiffness}

This step deals with the identification of the different sub-matrices of $\mathbf{M}, \mathbf{D}$ and $\mathbf{K}$ written as $\mathbf{S}$ in (5)

$$
\mathbf{M}=\left[\begin{array}{ll}
\mathbf{M}_{\mathbf{\Gamma}} & \mathbf{M}_{\mathbf{c}} \\
\mathbf{M}_{\mathbf{c}}^{T} & \mathbf{M}_{\boldsymbol{\Omega}}
\end{array}\right], \quad \mathbf{D}=\left[\begin{array}{ll}
\mathbf{D}_{\mathbf{\Gamma}} & \mathbf{D}_{\mathbf{c}} \\
\mathbf{D}_{\mathbf{c}}^{T} & \mathbf{D}_{\boldsymbol{\Omega}}
\end{array}\right], \quad \mathbf{K}=\left[\begin{array}{cc}
\mathbf{K}_{\boldsymbol{\Gamma}} & \mathbf{K}_{\mathbf{c}} \\
\mathbf{K}_{\mathbf{c}}^{T} & \mathbf{K}_{\mathbf{\Omega}}
\end{array}\right]
$$

These sub-matrices are taken as $\mathbf{M}_{\mathbf{c}}=0$ and $\mathbf{D}_{\Omega}, \mathbf{K}_{\boldsymbol{\Omega}}, \mathbf{M}_{\Omega}$ are diagonal, the last one being the identity matrix: $\mathbf{K}_{\Omega}=\mathbf{k}_{\Omega}, \mathbf{D}_{\Omega}=\mathbf{d}_{\Omega}, \mathbf{M}_{\Omega}=\mathbf{I}_{\Omega}$. Obtaining the components of all sub-matrices is performed by identifying the reduced form of $\mathbf{S}$, expressed with the sub-component of matrices $\mathbf{M}, \mathbf{D}$ and $\mathbf{K}$, with the terms coming from the partial fraction decomposition of $\mathbf{N} / q$. 
The expanded expression of (7) becomes

$$
\begin{gathered}
\hat{\mathbf{z}}=(\mathrm{j} \omega)^{2} \mathbf{M}_{\mathbf{\Gamma}}+\mathbf{j} \omega \mathbf{D}_{\mathbf{\Gamma}}+\mathbf{K}_{\mathbf{\Gamma}}-\sum_{l=1}^{n_{\Omega}} \mathbf{D}_{\mathbf{c}}^{l} \mathbf{D}_{\mathbf{c}}^{I T} \\
-\sum_{l=1}^{n_{\Omega}} \mathrm{j} \omega \frac{\mathbf{D}_{\mathbf{c}}^{l} \mathbf{K}_{\mathbf{c}}^{l^{T}}+\mathbf{K}_{\mathbf{c}}^{l} \mathbf{D}_{\mathbf{c}}^{l T}-d_{l} \mathbf{D}_{\mathbf{c}}^{l} \mathbf{D}_{\mathbf{c}}^{l T}}{-\omega^{2}+\mathbf{j} \omega d_{l}+k_{l}}-\sum_{l=1}^{n_{\Omega}} \frac{\mathbf{K}_{\mathbf{c}}^{l} \mathbf{K}_{\mathbf{c}}^{l T}-k_{l} \mathbf{D}_{\mathbf{c}}^{l} \mathbf{D}_{\mathbf{c}}^{l T}}{-\omega^{2}+\mathbf{j} \omega d_{l}+k_{l}}
\end{gathered}
$$

where matrices $\mathbf{D}_{\mathbf{c}}^{l}$, $\mathbf{K}_{\mathbf{c}}^{l}$ are the lth column vectors of $\mathbf{D}_{\mathbf{c}}, \mathbf{K}_{\mathbf{c}}$. The rational fraction $\mathbf{N} / q$ can be written as a sum of the Euclidean division of all terms of $\mathbf{N} / q$ and the decomposition of the rational fraction of the remaining part

$$
\begin{gathered}
\mathbf{N} / q=(\mathrm{j} \omega)^{2} \mathbf{R}_{-2}+\mathbf{j} \omega \mathbf{R}_{-1}+\mathbf{R}_{\mathbf{0}}+\sum_{l=1}^{n_{q}} \frac{\mathbf{R}_{l}}{\mathrm{j} \omega-p_{l}} \\
=(\mathbf{j} \omega)^{2} \mathbf{R}_{-2}+\mathbf{j} \omega \mathbf{R}_{-1}+\mathbf{R}_{\mathbf{0}}+\sum_{l=1}^{n_{q} / 2} \frac{2 \mathrm{j} \omega \operatorname{Re}\left(\mathbf{R}_{l}\right)-2 \operatorname{Re}\left(\mathbf{R}_{l} p_{l}^{*}\right)}{-\omega^{2}-2 \mathrm{j} \omega \operatorname{Re}\left(p_{l}\right)+\left\|p_{l}\right\|^{2}}
\end{gathered}
$$

where $p_{l}$ are the poles and $\mathbf{R}_{l}$ the associated residues of the rational fraction. Writing that $\hat{\mathbf{Z}}$ must be equal to $\mathbf{N} / q$ leads to three coupled systems of equations on sub-matrices $\mathbf{M}_{\Gamma}, \mathbf{D}_{\Gamma}, \mathbf{K}_{\Gamma}, \mathbf{D}_{\Omega}, \mathbf{K}_{\Omega}, \mathbf{D}_{\mathbf{c}}$ and $\mathbf{K}_{\mathbf{c}}$

$$
\begin{aligned}
& \begin{cases}\mathbf{M}_{\Gamma}=\mathbf{R}_{-2} & \\
\mathbf{D}_{\Gamma}=\mathbf{R}_{-1} & \\
d_{l}=-2 \operatorname{Re}\left(p_{l}\right), & 1 \leq l \leq n_{\Omega} \\
k_{l}=\left\|p_{l}\right\|^{2}, & 1 \leq l \leq n_{\Omega}\end{cases} \\
& \left\{\begin{array}{l}
\mathbf{D}_{\mathbf{c}}^{l} \mathbf{K}_{\mathbf{c}}^{l T}+\mathbf{K}_{\mathbf{c}}^{l} \mathbf{D}_{\mathbf{c}}^{I T}-d_{l} \mathbf{D}_{\mathbf{c}}^{l} \mathbf{D}_{\mathbf{c}}^{l T}=-2 \operatorname{Re}\left(\mathbf{R}_{l}\right) \\
\mathbf{K}_{\mathbf{c}}^{l} \mathbf{K}_{\mathbf{c}}{ }_{\mathbf{c}}-k_{l} \mathbf{D}_{\mathbf{c}}^{l} \mathbf{D}_{\mathbf{c}}^{l T}=2 \operatorname{Re}\left(\mathbf{R}_{l} p_{l}^{*}\right) \\
1 \leq l \leq n_{\Omega}
\end{array}\right. \\
& \left\{\mathbf{K}_{\boldsymbol{\Gamma}}=\mathbf{R}_{\mathbf{0}}+\sum_{l=1}^{n_{q}} \mathbf{D}_{\mathbf{c}}^{l} \mathbf{D}_{\mathbf{c}}^{l T}\right.
\end{aligned}
$$

$\mathbf{M}_{\boldsymbol{\Gamma}}, \mathbf{D}_{\boldsymbol{\Gamma}}, d_{l}$ and $k_{l}$ are obtained directly from the first problem (B.4). The last system of equations produces $\mathbf{K}_{\boldsymbol{\Gamma}}$ as soon as $\mathbf{D}_{\mathbf{c}}, \mathbf{K}_{\mathbf{c}}$ are known and the crucial point is in solving the second set of equations producing $\mathbf{D}_{\mathbf{c}}, \mathbf{K}_{\mathbf{c}}$.

It can be solved, as proposed in [13], by using the Takagi decomposition [53] of $\mathbf{R}_{l}$. The Takagi decomposition can be effected by using the method described in [54].

\section{Appendix C. Construction of the stochastic impedance}

This section recalls briefly the main properties of the probabilistic model developed by Soize $[7,8]$.

\section{C.1. The set of positive-definite random matrices}

Let us consider the "mean value" of one of the mass, damping and stiffness matrices, denoted by A. The random values of this quantity are denoted by $\overline{\mathbf{A}}$. They are within the set of positive-definite random matrices, denoted as $S E^{+}$. This set contains the matrices which have the following properties:

$$
\begin{cases}\left.\overline{\mathbf{A}} \in \mathbb{M}^{+}(\mathbb{R})\right) & \\ E\left\{\|\overline{\mathbf{A}}\|_{F}^{2}\right\}<+\infty & \text { second }- \text { order random variable } \\ E\{\overline{\mathbf{A}}\}=\mathbf{A} & \text { mean value is matrix } \mathbf{A} \\ E\{\ln (\operatorname{det}(\overline{\mathbf{A}}))\}<+\infty & \end{cases}
$$

where $\mathbb{M}^{+}(\mathbb{R})$ ) is the set of positive definite matrices. The last constraint yields that the inverse of $\bar{A}$ is a second-order random variable, so $E\left\{\left\|\overline{\mathbf{A}}^{-1}\right\|_{F}^{2}\right\}<+\infty$.

Since $\mathbf{A}$ is a positive-definite deterministic matrix, there is a positive definitive upper triangular matrix $\mathbf{L}$ such that $\mathbf{A}=\mathbf{L}^{T} \mathbf{L}$ and the ensemble $S E^{+}$is also the set of random matrices $\overline{\mathbf{A}}$ which are written as

$$
\overline{\mathbf{A}}=\mathbf{L}^{T} \overline{\mathbf{G}} \mathbf{L}
$$

in which $\overline{\mathbf{G}}$ has the same properties as $\mathbf{A}$, but has a probabilistic mean which is equal to a unit matrix. This new set of normalized positive-definite random matrices is denoted by $S G^{+}$. 


\section{C.2. The set of normalized positive-definite random matrices}

The set of normalized positive-definite random matrices is defined as a set of random matrices $\overline{\mathbf{G}}$ with values in $\mathbb{R}$ verifying

$$
\begin{cases}\overline{\mathbf{G}} \in \mathbb{M}^{+}(\mathbb{R}) & \\ E\left\{\|\overline{\mathbf{G}}\|_{F}^{2}\right\}<+\infty & \text { second - order random variable } \\ E\{\overline{\mathbf{G}}\}=\mathbf{I}_{\mathbf{n}} & \text { mean value is identity matrix } \\ E\{\ln (\operatorname{det}(\overline{\mathbf{G}}))\}=\nu_{G} & \text { (where } \left.\left|\nu_{G}\right|<+\infty\right)\end{cases}
$$

The value of $\nu_{G}$ can be considered as a scattering parameter $\delta$ which is a measure of the dispersion of the probability model of the random matrix $\overline{\mathbf{G}}$ around the mean value $\mathbf{I}_{\mathbf{n}} . \delta$ is a real parameter which must comply with

$$
\begin{aligned}
0<\delta & <\left\{\frac{E\left\{\left\|\overline{\mathbf{G}}-\mathbf{I}_{\mathbf{n}}\right\|_{F}^{2}\right\}}{\left\|\mathbf{I}_{\mathbf{n}}\right\|_{F}^{2}}\right\}^{1 / 2} \\
& =\frac{1}{n} E\left\{\left\|\overline{\mathbf{G}}-\mathbf{I}_{\mathbf{n}}\right\|_{F}^{2}\right\}^{1 / 2}
\end{aligned}
$$

To ensure that the fourth condition is verified, $\delta$ should be chosen independent of $n$ and such that

$$
0<\delta<\sqrt{\frac{n+1}{n+5}}
$$

Several procedures can be used for choosing $\delta$ [12,7]. In this study, it is determined by using the level of data scattering of ground elastic properties usually taken into account by engineers.

\section{C.3. Construction of the unit random matrices}

As $\overline{\mathbf{G}}$ is positive-definite, it can be written as $\overline{\mathbf{G}}=\overline{\mathbf{L L}}^{T}$ by Cholesky's factorization. Let us introduce $\sigma_{n}=\delta / \sqrt{n+1}$. Using the maximum entropy principle, it can been shown [8] that the different probability density functions related to the coefficients $\overline{\mathbf{L}}_{i j}$ of the triangular matrix comply with the following properties:

- random variables $\overline{\mathbf{L}}_{i j}, i \leq j$ are independent,

- for $i<j, \overline{\mathbf{L}}_{i j}=\sigma_{n} U_{i j}$ where $U_{i j}$ is a Gaussian random variable with zero mean and unit variance,

- for $i=j, \overline{\mathbf{L}}_{i j}=\sigma_{n} \sqrt{2 V_{i}}$ where $V_{i}$ is a Gamma random variable with positive real values. Its density function is given in [7].

\section{References}

[1] T. Dawn, C. Stanworth, Ground vibrations from passing trains, Journal of Sound and Vibration 66 (3) (1979) $355-362$.

[2] J. Melke, Noise and vibration from underground railway lines: proposals for a prediction procedure, Journal of Sound and Vibration 120 (2) (1986) 391-406.

[3] H. Hunt, Modelling of rail vehicles and track for calculation of ground-vibration transmission into buildings, Journal of Sound and Vibration 193 (1) (1995) 185-194.

[4] O. Hassan, Transmission of structure-borne sound in buildings above railway tunnels, Building Acoustics 8 (4) (2001) $269-299$.

[5] C. Guigou-Carter, M. Villot, B. Guillerme, C. Petit, Analytical and experimental study of sleeper SAT S 312 in slab track Sateba system, Journal of Sound and Vibration 293 (3) (2006) 878-887.

[6] C. Esveld, Modern Railway Track, MRT-Production, Zaltbommel, 1989.

[7] C. Soize, Maximum entropy approach for modeling random uncertainties in transient elastodynamics, Journal of the Acoustical Society of America 109 (5) (2001) 1979-1996.

[8] C. Soize, A nonparametric model of random uncertainties for reduced matrix models in structural dynamics, Probabilistic Engineering Mechanics 15 (3) (2000) 277-294.

[9] J.P. Wolf, Consistent lumped-parameter models for unbounded soil: frequency-independent stiffness, damping and mass matrices, Earthquake Engineering and Structural Dynamics 20 (1991) 33-41.

[10] J.P. Wolf, Foundation Vibration Analysis Using Simple Physical Models, Prentice Hall, NJ, 1994.

[11] X. Du, M. Zhao, Stability and identification for rational approximation of frequency response function of unbounded soil, Earthquake Engineering and Structural Dynamics 39 (2009) 165-186.

[12] R. Cottereau, Probabilistic Models of Impedance Matrices, PhD Thesis, Ecole Centrale Paris, 2006.

[13] R. Cottereau, D. Clouteau, C. Soize, Construction of a probabilistic model for impedance matrices, Computer Methods in Applied Mechanics and Engineering 197 (2007) 2252-2268.

[14] J. Mondot, B. Petersson, Characterization of structure-borne sound sources: the source descriptor and the coupling function, Journal of Sound and Vibration 114 (3) (1987) 507-518.

[15] A.T. Moorhouse, On the characteristic power of structure-borne sound sources, Journal of Sound and Vibration 248 (3) (2001) $441-459$.

[16] B. Petersson, J. Plunt, On effective mobilities in the prediction of structure-borne sound transmission between a source structure and a receiving structure. Part I. Theoretical background and basic experimental studies, Journal of Sound and Vibration 82 (4) (1982) $517-529$.

[17] B. Petersson, A thin-plates model for the moment mobility at the intersection of two perpendicular plates, Journal of Sound and Vibration 108 (3) (1986) 471-485.

[18] S. Yap, The Role of Moments and Forces in Structure-Borne Sound Emission from Machines in Buildings, PhD Thesis, University of Liverpool, 1998. 
[19] P. Ropars, M. Villot, P. Jean, Calcul 2D de la puissance vibratoire d'origine ferroviaire injectée dans un bâtiment par la méthode des mobilités (2-D calculation of railway vibrational power injected in a building by the mobility method), 10éme Congrés Français d'Acoustique, Lyon, France, 2010.

[20] B. Petersson, B.M. Gibbs, Use of the source descriptor concept in studies of multi-point and multi-directional vibrational sources, Journal of Sound and Vibration 168 (1) (1993) 157-176.

[21] L. Cremer, M. Heckl, U. Hungar, Structure-borne Sound Structural Vibrations and Sound Radiation at Audio Frequencies, Springer-Verlag, Berlin, Heidelberg, New York, 1973.

[22] M. Ohlrich, Structure-borne sound sources and their power transfer, International Congress of Noise Control Engineering, La Haye, Holland, 2001, pp. 2116-2127.

[23] Y. Koh, R. White, Analysis and control of vibrational power transmission to machinery supporting structures subjected to a multi-excitation system. Part I. Driving point mobility matrix of beams and rectangular plates, Journal of Sound and Vibration 196 (4) (1996) $469-493$.

[24] P. Ropars, Modélisation des vibrations d'origine ferroviaire transmises aux bâtiments par le sol (Modeling of Vibration Induced by Railway Transmitted to Buildings from the Soil), PhD Thesis, Université Paris-Est Marne la Vallée, 2011.

[25] P. Jean, Boundary and finite elements for 2D soil-structure interaction problems, Acta Acustica 87 (1) (2001) 56-66.

[26] P. Jean, C. Guigou, M. Villot, A 2.5D BEM model for ground-structure interaction, Building Acoustics 11 (3) (2004) 1-17.

[27] Y.B. Yang, H.H. Hung, Wave Propagation for Train-Induced Vibrations, A Finite/Infinite Element Approach, World Scientific Publishing, Singapore, 2009.

[28] J. O'Brien, D.C. Rizos, A 3D BEM-FEM methodology for simulation of high speed train induced vibrations, Soil Dynamics and Earthquake Engineering 25 (4) (2005) 289-301.

[29] M. Mohammadi, D.L. Karabalis, Dynamic 3-D soil-railway track interaction by BEM-FEM, Earthquake Engineering and Structural Dynamics 24 (9) (1995) 1177-1193.

[30] M. Villot, P. Ropars, E. Bongini, F. Poisson, Modelling the influence of structural modifications on the response of a building to railway vibration, Noise Control Engineering Journal 59 (6) (2011) 641-651.

[31] M. Arnst, Three-Dimensional Modelling of Free Field and Structural Vibration due to Harmonic and Transient Loading in a Tunnel, PhD Thesis, Ecole Centrale Paris \& Faculteit Toegepaste Wetenschappen, 2003.

[32] H. Chebli, R. Othman, D. Clouteau, M. Arnst, G. Degrande, 3D periodic BE-FE model for various transportation structures interacting with soil, Computers and Geotechnics 35 (2008) 22-32.

[33] R. Craik, Sound Transmission through buildings using Statistical Energy Analysis, Gower Press, Aldershot, 1996.

[34] I. Bosmans, G. Vermeir, P. Mees, Coupling loss factors for coupled anisotropic plates, Journal of Sound and Vibration 250 (2) (2002) $351-355$.

[35] M. Villot, P. Ropars, P. Jean, Modeling a building response to railway vibration using a source-receiver approach, International Conference on Structural Dynamics, Leuven, Belgium, 2011, pp. 671-675.

[36] P. Mees, O. Vermeir, Structure-borne sound transmission at elastically connected plates, Journal of Sound and Vibration 166 (1) (1993) 55-76.

[37] I. Bosmans, P. Mees, G. Vermeir, Structure-Borne sound transmission between thin orthotropic plates: analytical solutions, Journal of Sound and Vibration 191 (1) (1996) 75-90.

[38] Norm 1, Ground-Borne Noise and Vibration Arising from Rail Systems, ISO 14837-1.

[39] Norm 2, Vibration and Shock Measurement of Vibration in Buildings from Landbased Transport and Guidance to Evaluation of its Effects on Human Beings, Norwegian Standard NS 8176.E second edition.

[40] C. Sanathanan, J. Koerner, Transfer function synthesis as a ratio of two complex polynomials, IEEE Transactions on Automatic Control (1963) $56-58$.

[41] C. Soize, R. Ganem, Physical systems with random uncertainties: chaos representations with arbitrary probability measure, SIAM Journal on Scientific Computing 26 (2) (2004) 395-410.

[42] M. Laviolette, J. Seaman Jr., J. Douglas Barrett, W. Woodall, A probabilistic and statistical view of fuzzy methods, Technometrics 37 (3) (1995) $249-261$.

[43] D.M. Ghiocel, R.G. Ghanem, Stochastic finite-element analysis of seismic soil-structure interaction, Journal of Engineering Mechanics 128 (1) (2002) 66-77.

[44] G.A. Fenton, Estimation for stochastic soil models, Journal of Geotechnical and Geoenvironmental Engineering 125 (6) (1999) $470-485$.

[45] M. Schevenels, G. Lombaert, G. Degrande, D. Degrauwe, B. Schoors, The Green's functions of a vertically inhomogeneous soil with a random dynamic shear modulus, Probabilistic Engineering Mechanics 22 (1) (2007) 100-111.

[46] S. Jones, K. Kuo, M. Hussein, H. Hunt, Prediction uncertainties and inaccuracies resulting from common assumptions in modelling vibration from underground railways, Journal of Rail and Rapid Transit 226 (5) (2012) 501-512.

[47] R. Ghanem, P. Spanos, Stochastic Finite Elements: A Spectral Approach, Springer-Verlag, New York, 1991.

[48] R. Cottereau, D. Clouteau, C. Soize, Modèle dynamique équivalent de matrices d'impédance de fondation (Equivalent dynamic model of foundation impedance matrix), 8éme Colloque National en Calcul des Structures, Chatenay Malabry, France, 2007.

[49] V. Kharitonov, On a generalization of a stability criterion, Seria Fiziko-Mathematicheskaia 1 (1978) 53-57.

[50] M. Powell, Direct search algorithms for optimization calculations, Acta Numerica 7 (1998) 287-336.

[51] Norm 3, Normes NF-P 06-13, Règles de construction parasismique, Règles PS applicables aux bâtiments (Seismic building rules, PS rules for buildings), - PS92, Eyrolles Deuxième edition, 1998.

[52] W.-H. Wu, W.-H. Lee, Systematic lumped-parameter models for foundations based on polynomial-fraction approximation, Earthquake Engineering and Structural Dynamics 31 (2002) 1383-1412.

[53] T. Takagi, On an algebraic problem related to an analytic theorem of Caratheodory and Fejer and on an allied theorem of Landau, Japanese Journal of Mathematics 1 (1925) 83-93.

[54] W. Xiaohong, Q. Sanzheng, A parallel Jacobi method for the Takagi factorization, International Conference on Parallel and Distributed Processing Techniques and Application, Las Vegas, USA, 2002, pp. 206-221. 